\section{Geometric and arithmetic realized comoments}

Geometric and arithmetic comoments

\author{
Kwangil Bae \\ Chonnam National University, Gwangju, Republic of Korea
}

\begin{abstract}
The author investigates realized comoments that overcome the drawback of conventional ones and derive the following findings. First, the author proves that (even generalized) geometric implied lower-order comoments yield neither geometric realized third comoment nor fourth moment. This is in contrast to previous studies that produce geometric realized third moment and arithmetic realized higher-order moments through lower-order implied moments. Second, arithmetic realized joint cumulants are obtained through complete Bell polynomials of lower-order joint cumulants. This study's realized measures are unbiased estimators and they can, therefore, overcome the drawbacks of conventional realized measures.
\end{abstract}

Keywords Realized joint cumulants, Realized comoments, Log returns, Implied moments,

Aggregation property

Paper type Research paper

\section{Introduction}

The framework suggested by Andersen et al. (2003) produces low-frequency variance from high-frequency returns. This so-called realized variance is defined as a sum of squares of subperiodical returns. Kraus and Litzenberger (1976) and Dittmar (2002) demonstrate the relationship between higher-order moments and expected returns, and the concept of the realized variance has been extended to realized higher-order moments. In many studies, including those of Amaya et al. (2015), Sim (2016), Kim (2016), Mei et al. (2017), Kinateder and Papavassiliou (2019), and Ahmed and Al Mafrachi (2021) [1], a realized kth order moment is defined as a sum of $k$ th orders of sub-periodical returns. However, according to Amaya $e t a l$. (2015) and Bae and Lee (2021), these conventional realized higher-order moments can reflect neither the volatility of volatility nor cross-period relation among sub-periodical returns and are, therefore, flawed. Several studies attempt to resolve these problems by providing unbiased realized moments, and such research is summarized in Table 1.

The revised realized moments are developed based on Neuberger's (2012) Aggregation Property, through which the author presents arithmetic and geometric realized third moments using changes in prices and implied variances [2]. Bae and Lee (2021) extend the arithmetic realized moments in two folds. One is the extension of moments to comoments, and the other is an extension of the order from three to four. Furthermore, Fukasawa and

\section{JEL Classification — G11, G12, G13}

(C) Kwangil Bae. Published in Journal of Derivatives and Quantitative Studies: 선물연구. Published by Emerald Publishing Limited This article is published under the Creative Commons Attribution (CC BY 4.0) licence. Anyone may reproduce, distribute, translate and create derivative works of this article (for both commercial and non-commercial purposes), subject to full attribution to the original publication and authors. The full terms of this licence maybe seen at http://creativecommons.org/licences/by/4.0/ legalcode.

The author is grateful for the 2021 financial assistance provided by The Research Foundation, Graduate School of Business, Chonnam National University, Republic of Korea. The author is also grateful to the anonymous referees, Jun-Kee Jeon, Hyoung-Goo Kang, Jangkoo Kang, Hwa-Sung Kim, Hyeng-Keun Koo, Soonhee Lee, Sun-Joong Yoon and the editors (Sol Kim and Eun Jung Lee) for valuable and detailed comments. All errors are the authors' responsibility.

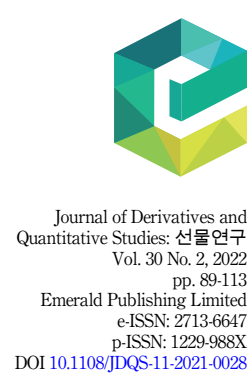

Received 5 November 2021 Revised 21 January 2022 Accepted 24 January 2022 
JDQS

30,2

90

Matsushita (2021) provide arithmetic moments of general orders. However, to the best of the author's knowledge, geometric comoments, geometric moments above the third order and arithmetic comoments above the fourth order have not yet been developed.

The current study attempts to complete Table 1. Our first target is the geometric realized moments and comoments. Many financial studies use geometric returns (log-returns) because they have useful features such as time-additivity. Accordingly, Neuberger (2012) proposes geometric realized third moment. To find the missing geometric measures in the aforementioned table, we extend information set to include lower order moments because all the revised moments are obtained through the lower order moments. However, unlike the aforementioned studies, the current research demonstrates that (even generalized) implied variance and covariance do not yield realized third comoment, although they yield realized covariance. Moreover, we reveal that (even generalized) implied third moment does not yield realized fourth moments.

Our second target is the arithmetic realized comoments for general orders. We previously mentioned the usefulness of the log-returns, and as shown in Table 1, arithmetic realized comoments up to the fourth-order are developed. However, arithmetic returns are also as wellused as geometric returns, and financial studies require the estimation of higher-order comoments. For example, Rubinstein (1973) extends the traditional Capital Asset Pricing Model (CAPM)

$$
E\left[r_{i}\right]=r_{f}+\lambda E\left[\left(r_{M}-E\left[r_{M}\right]\right)\left(r_{i}-E\left[r_{i}\right]\right)\right]
$$

with $\lambda=\frac{1}{\sigma_{M}^{2}} E\left[r_{M}-r_{f}\right]$ to

$$
E\left[r_{i}\right]=r_{f}+\sum_{l=2}^{\infty} \lambda_{l} E\left[\left(r_{M}-E\left[r_{M}\right]\right)^{l-1}\left(r_{i}-E\left[r_{i}\right]\right)\right],
$$

and Chung et al. (2006) and Hung (2008) demonstrate that comoments above the fourth order are priced. Accordingly, we attempt to identify the realized comoment above the fourth-order under the arithmetic sense. To do so, we extend Fukasawa and Matsushita's (2021) arithmetic realized cumulants [3]. While Neuberger (2012) and Bae and Lee (2021) attempt to obtain all functions satisfying the Aggregation Property given information set, Fukasawa and Matsushita (2021) present a rule among realized cumulants. Adopting their methodology, we obtain arithmetic realized joint cumulants through complete Bell polynomials of lower-order joint cumulants. Our realized measures are unbiased estimators and they can, therefore, overcome the drawbacks of conventional realized measures.

The rest of the paper is organized as follows. Neuberger's (2012) Aggregation Property is reviewed, and generalized geometric moments are defined in section 2 . The non-existence of geometric higher order moments and comoments is demonstrated in section 3. Joint

\begin{tabular}{lll}
\hline Order & Arithmetic realized moments & Geometric realized moments \\
\hline \multicolumn{2}{l}{ Panel A. Realized moments } & \\
3 & Neuberger (2012) & Neuberger (2012) \\
4 & Bae and Lee (2021) & - \\
Above 4 & Fukasawa and Matsushita (2021) & - \\
Panel B. Realized comoments & - \\
3 & Bae and Lee (2021) & - \\
4 & Bae and Lee (2021) & - \\
Above 4 & - &
\end{tabular}

Table 1.

Revised realized moments and comoments

\begin{abstract}
$$
\text { geometric higher order moments and comoments is demonstrated in section } 3 \text {. Joint }
$$
\end{abstract}


cumulants are explained and arithmetic realized joint cumulants outlined in section 4. Finally, concluding remarks are presented in section 5.

\section{Preliminary: aggregation property and generalized geometric realized moments}

Consider a martingale process $S_{t}$ and a partition $\left\{t_{0}, t_{1}, \ldots, t_{N}\right\}$ on $[0, T]$ such that $0=t_{0} \leq t_{1} \leq t_{2} \leq \ldots \leq t_{N}=T$. Equation (1) holds for $k=2$.

$$
E_{0}\left[\left(S_{T}-S_{0}\right)^{k}\right]=E_{0}\left[\sum_{j=1}^{N}\left(S_{t_{j}}-S_{t_{j-1}}\right)^{k}\right]
$$

Owing to this relation, $\sum_{j=1}^{N}\left(S_{t_{j}}-S_{t_{j-1}}\right)^{2}$ is referred to as realized second moment or realized variance. However, Equation (1) does not hold for the higher-order $(k \geq 3)$, which makes obtaining realized higher-order moments non-straightforward. To solve this problem, Neuberger (2012) proposes the aggregation property that generalizes Equation (1) as follows.

\section{Definition 2.1. Aggregation property}

Let $X=\left(X_{t}, 0 \leq t \leq T\right)$ be an adapted vector-valued stochastic process defined on a filtration. A function $g$ on a vector-valued process $X$ satisfies the $A P$ (aggregation property) if

$$
E_{r}\left[g\left(X_{u}-X_{r}\right)\right]=E_{r}\left[g\left(X_{u}-X_{t}\right)\right]+E_{r}\left[g\left(X_{t}-X_{r}\right)\right], \forall(r, t, u) 0 \leq r \leq t \leq u \leq T .
$$

Owing to the law of the iterated expectations, when a function $g$ satisfies the AP, we have

$$
E_{0}\left[g\left(X_{T}-X_{0}\right)\right]=E_{0}\left[\sum_{j=1}^{N} g\left(X_{t_{j}}-X_{t_{j-1}}\right)\right] .
$$

In this regard, $\sum_{j=1}^{N} g\left(X_{t_{j}}-X_{t_{j-1}}\right)$ can be called a realized $E_{0}\left[g\left(X_{T}-X_{0}\right)\right]$.

To develop the realized moments of $\log$ returns, $X_{t}$ needs to contain $\log$ prices $s_{t}=\ln S_{t}$, and additional arguments can contribute to constructing the abundant functions that satisfy the AP. For example, Neuberger (2012) uses $\Delta s$ and $\Delta v^{N}$ that are changes in log price $s_{t}$ and specific generalized variance $v_{t}^{N}$, respectively. Furthermore, he demonstrates that $e^{\Delta s}-1, \Delta s$, $\Delta v^{N}, e^{\Delta s}\left(\Delta v^{N}+2 \Delta s\right)$, and their linear combination satisfy the AP when the stock price $S_{t}$ is a martingale. Thus, the following form satisfies the AP.

$$
\begin{aligned}
g^{N}\left(\Delta s, \Delta v^{N}\right) & =-12\left(e^{\Delta s}-1\right)+6 \Delta s-3 \Delta v^{N}+3 e^{\Delta s}\left(\Delta v^{N}+2 \Delta s\right) \\
& =3 \Delta v^{N}\left(e^{\Delta s}-1\right)+6\left(\Delta s e^{\Delta s}-2 e^{\Delta s}+\Delta s+2\right)
\end{aligned}
$$

Moreover, the martingale property yields $E_{t}\left[g^{N}\left(\ln S_{T}-\ln S_{t}, v_{T}^{N}-v_{t}^{N}\right)\right]=E_{t}\left[K\left(\ln S_{T}-\ln S_{t}\right)\right]$ for $K(x)=6\left(x e^{x}-2 e^{x}+x+2\right)=x^{3}+O\left(x^{4}\right)$. Thus, Neuberger (2012) refers to,

$$
\sum_{j=1}^{N} g^{N}\left(\ln S_{j}-\ln S_{j-1}, v_{t_{j}}^{N}-v_{t_{j-1}}^{N}\right)
$$

as a realized third moment of $\log$ return $\ln S_{T}-\ln S_{0}$. However, the study presents neither any realized comoments nor realized fourth moments. It may be resolved by additional information of their lower-order implied comoments of log returns. According to Neuberger (2012), implied variance contributes to constructing the realized third moment for both arithmetic and log returns. Similarly, Bae and Lee (2021) show that realized comoments for the arithmetic returns
Geometric and arithmetic comoments 
require lower-order moments and comoments. Thus, implied covariance and variances of log returns may contribute to the realized third comoment of log returns, and implied variance and third moment of log returns may contribute to the realized fourth moment of log returns.

To consider covariation, we use two martingale processes $S_{1, t}$ and $S_{2, t}$, and their log values are $s_{1, t}$ and $s_{2, t}$, respectively. For the variant functions satisfying the AP, we allow flexibility on the forms of implied comoments, and we define generalized comoments as follows [4].

\section{Definition 2.2. (Implied) generalized $(k, l)$-comoment}

We refer to $E_{t}\left[f^{k, l}\left(s_{1, T}-s_{1, t}, s_{2, T}-s_{2, t}\right)\right]$ as a generalized $(k, l)$-comoment at time $t$ when $f^{k, l}$ is an analytic function such that $\frac{f^{k, l}(a, b)}{a^{k} b^{l}} \rightarrow 1$ as $(a, b) \rightarrow(0,0)$. For convenience, we call it a generalized $(k+l)$-moment and replace $f^{k, l}$ with $f^{k+l}$ if $k$ or $l$ is zero.

Equipped with the above log prices processes and implied comoments, we investigate higher-order realized comoments. Consider a partitioned vector process $x=\left(s_{1}, s_{2}, m\right)$, where $m$ is a vector process of comoments. When a function $g$ satisfies

$$
E_{0}\left[\sum_{j=1}^{N} g\left(x_{t_{j}}-x_{t_{j-1}}\right)\right]=E_{0}\left[g_{r}^{k, l}\left(s_{1, T}-s_{1,0}, s_{2, T}-s_{2,0}\right)\right]
$$

with a function $g_{r}^{k, l}(\cdot, \cdot)$ such that

$$
g_{r}^{k, l}\left(s_{1, T}-s_{1,0}, s_{2, T}-s_{2,0}\right) \approx\left(s_{1, T}-s_{1,0}\right)^{k}\left(s_{2, T}-s_{2,0}\right)^{l},
$$

$E_{0}\left[\sum_{j=1}^{N} g\left(x_{t_{j}}-x_{t_{j-1}}\right)\right]$ is close to ordinary comoment. Thus, a realized comoment is defined as follows.

Definition 2.3. Realized $(k, l)$-comoment

For a partitioned vector process $x=\left(s_{1}, s_{2}, m\right)$ including a vector process $m_{t}$, let us call

$$
\sum_{j=1}^{N} g\left(x_{t_{j}}-x_{t_{j-1}}\right)
$$

a realized $(k, l)$-comoment if a function $g$ satisfies the AP and is decomposed as follows

$$
g\left(x_{\tau}-x_{t}\right)=\phi\left(x_{\tau}-x_{t}\right)+g_{r}^{k, l}\left(s_{1, \tau}-s_{1, t}, s_{2, \tau}-s_{2, t}\right),
$$

where $\phi$ is a function that satisfies $E_{t}\left[\phi\left(x_{T}-x_{t}\right)\right]=0$, and $g_{r}^{k, l}$ is a function such that that $\frac{g_{r}^{k, l}(a, b)}{a^{k} b^{l}} \rightarrow 1$ as $(a, b) \rightarrow(0,0)$. For convenience, we refer to Equation (8) as a realized $(k+l)$ moment if $k$ or $l$ is zero.

Note that when a function $g$ satisfies the AP and has the decomposition in Equation (9), we have

$$
E_{0}\left[\sum_{j=1}^{N} g\left(x_{t_{j}}-x_{t_{j-1}}\right)\right]=E_{0}\left[g\left(x_{T}-x_{0}\right)\right]=E_{0}\left[g_{r}^{k, l}\left(s_{1, T}-s_{1,0}, s_{2, T}-s_{2,0}\right)\right] .
$$

Thus, it is close to the standard comoment when $g_{r}^{k, l}\left(s_{1, T}-s_{1,0}, s_{2, T}-s_{2,0}\right) \approx$ $\left(s_{1, T}-s_{1,0}\right)^{k}\left(s_{2, T}-s_{2,0}\right)^{l}$. 


\section{Nonexistence of geometric realized higher-order comoments}

Based on Definition 2.2, we denote the generalized 2-moment for the asset $i \in\{1,2\}$ as $v_{i}$ with its underlying function $f^{2}(\cdot)$. In addition, let us denote the generalized comoment as $v_{c}$ with its underlying function $f^{1,1}(\cdot, \cdot)$. We first investigate the function satisfying the AP given the information set $x$ that includes $\log$ prices $\left(s_{1}, s_{2}\right)$, variances $\left(v_{1}, v_{2}\right)$ and covariance $\left(v_{c}\right)$ as follows.

Proposition 3.1. An analytic function $g$ satisfies the AP on the vector valued process $x=\left(s_{1}, s_{2}, v_{1}, v_{2}, v_{c}\right)$ if and only if $g$ is represented as follows:

$$
\begin{aligned}
g\left(\Delta s_{1}, \Delta s_{2}, \Delta v_{1}, \Delta v_{2}, \Delta v_{c}\right)= & h_{1}\left(e^{\Delta s_{1}}-1\right)+h_{2} \Delta s_{1}+h_{3}\left(e^{\Delta s_{2}}-1\right)+h_{4} \Delta s_{2} \\
& +h_{5} \Delta v_{1}+h_{6} \Delta v_{2}+h_{7} \Delta v_{c}+h_{8}\left(\Delta v_{1}-2 \Delta s_{1}\right)^{2} \\
& +h_{9}\left(\Delta v_{2}-2 \Delta s_{2}\right)^{2}+h_{10}\left(\Delta v_{1}-2 \Delta s_{1}\right)\left(\Delta v_{2}-2 \Delta s_{2}\right) \\
& +h_{11} e^{\Delta s_{1}}\left(2 \Delta v_{c}-\Delta v_{2}+2 \Delta s_{2}\right)+h_{12} e^{\Delta s_{2}}\left(2 \Delta v_{c}-\Delta v_{1}+2 \Delta s_{1}\right) \\
& +h_{13} e^{\Delta s_{1}}\left(\Delta v_{1}+2 \Delta s_{1}\right)+h_{14} e^{\Delta s_{2}}\left(\Delta v_{2}+2 \Delta s_{2}\right)
\end{aligned}
$$

for some constants $h_{1}, \cdots, h_{14}$, which satisfy one of the following five conditions:

(1) $h_{12}=h_{13}=h_{14}=0, f^{1,1}\left(\Delta s_{1}, \Delta s_{2}\right)=\Delta s_{2}\left(e^{\Delta s_{1}}-1\right)$ and $f^{2}(\Delta s)=2\left(e^{\Delta s}-\Delta s-1\right)$,

(2) $h_{11}=h_{13}=h_{14}=0, f^{1,1}\left(\Delta s_{1}, \Delta s_{2}\right)=\Delta s_{1}\left(e^{\Delta s_{2}}-1\right)$ and $f^{2}(\Delta s)=2\left(e^{\Delta s}-\Delta s-1\right)$,

(3) $h_{11}=h_{12}=h_{13}=h_{14}=0$ and $f^{2}(\Delta s)=2\left(e^{\Delta s}-\Delta s-1\right)$,

(4) $h_{8}=h_{9}=h_{10}=h_{11}=h_{12}=0$ and $f^{2}(\Delta s)=2\left(\Delta s e^{\Delta s}-\Delta s+1\right)$,

(5) $h_{8}=h_{9}=h_{10}=h_{11}=h_{12}=h_{13}=h_{14}=0$.

The proof is provided in Appendix 1.

Proposition 3.1 uses the information of implied covariance $v_{c}$ in addition to the variation of a single process in Neuberger (2012). It makes it possible to obtain new terms that satisfy the AP: the 10th term $\left(\Delta v_{1}-2 \Delta s_{1}\right)\left(\Delta v_{2}-2 \Delta s_{2}\right)$ with conditions (1), (2) and (3), the 11th term $e^{\Delta s_{1}}\left(2 \Delta v_{c}-\Delta v_{2}+2 \Delta s_{2}\right)$ with condition (1), and 12th term $e^{\Delta s_{2}}\left(2 \Delta v_{c}-\Delta v_{1}+2 \Delta s_{1}\right)$ with condition (2). These new terms are generalizations of $\left(\Delta v_{1}-2 \Delta s_{1}\right)^{2}$ and $e^{\Delta s_{1}}\left(\Delta v_{1}+2 \Delta s_{1}\right)$ observed in Neuberger (2012) in that the new terms become these when we set $S_{2, t}$ to be identical to $S_{1, t}$. The new terms may contribute to constructing new realized comoments, and Corollary 3.2 states the result.

Corollary 3.2. When the information set is given by $x=\left(s_{1}, s_{2}, v_{1}, v_{2}, v_{c}\right)$, there is not a realized (2,1)-comoment but a realized $(1,1)$-comoment.

The proof is in Appendix 1.

According to Corollary 3.2, we could not obtain realized (2,1)-comoment even when we have all its lower-order moment and comoment. This result is in contrast to Neuberger (2012) and Bae and Lee (2021), who obtain the realized third moment under both the arithmetic and log return and realized third comoment under the arithmetic return through their lower-order moments. Instead, Corollary 3.2 shows that $\Delta v_{c}$ with $\Delta v_{1}$ or $\Delta v_{2}$ produces the realized (1,1) comoment through

$$
\begin{aligned}
g\left(\Delta s_{1}, \Delta s_{2}, \Delta v_{2}, \Delta v_{c}\right) & =-\Delta v_{c}+\frac{1}{2} \Delta v_{2}+\frac{1}{2} e^{\Delta s_{1}}\left(2 \Delta v_{c}-\Delta v_{2}+2 \Delta s_{2}\right)-\Delta s_{2} \\
& =\left(e^{\Delta s_{1}}-1\right)\left(\Delta v_{c}-\frac{1}{2} \Delta v_{2}\right)+\left(e^{\Delta s_{1}}-1\right) \Delta s_{2},
\end{aligned}
$$

Geometric and arithmetic comoments 
JDQS 30,2 or

$$
g\left(\Delta s_{1}, \Delta s_{2}, \Delta v_{2}, \Delta v_{c}\right)=\left(e^{\Delta s_{2}}-1\right)\left(\Delta v_{c}-\frac{1}{2} \Delta v_{1}\right)+\left(e^{\Delta s_{2}}-1\right) \Delta s_{1} .
$$

Now, let us investigate functions satisfying the AP when the information includes higherorder moments for the single security. They are log price (s), implied second moment $\left(m_{2}\right)$ and implied third moment $\left(m_{3}\right)$, where the underlying function for the $k$ th moment is denoted by $f^{k}(\cdot)$.

Proposition 3.3. An analytic function $g$ on a vector valued process $x=\left(s, m_{2}, m_{3}\right)$ has the Aggregation Property on the vector valued process $x$ if and only if $g$ is represented as follows:

$$
\begin{aligned}
g\left(\Delta s, \Delta m_{2}, \Delta m_{3}\right)= & h_{1}\left(e^{\Delta s}-1\right)+h_{2} \Delta s+h_{3} \Delta m_{2}+h_{4} \Delta m_{3} \\
& +h_{5}\left(\Delta m_{2}+a \Delta m_{3}-2 \Delta s\right)^{2}+h_{6}\left(\Delta m_{2}+a \Delta m_{3}+2 \Delta s\right) e^{\Delta s}
\end{aligned}
$$

for some constants $h_{1}, \cdots, h_{6}$ and $a$, which satisfy one of the following three conditions:

(1) $h_{5}=h_{6}=0$.

(2) $h_{6}=0$ and $f^{2}(\Delta s)+a f^{3}(\Delta s)=2\left(e^{\Delta s}-\Delta s-1\right)$ for the constant $a$.

(3) $h_{5}=0$ and $f^{2}(\Delta s)+a f^{3}(\Delta s)=2\left(\Delta s e^{\Delta s}-e^{\Delta s}+1\right)$ for the constant $a$.

The proof is provided in Appendix 1.

Proposition 3.3 shows that three terms are satisfying the AP and containing $m_{3}$; the 4 th, 5 th and 6th terms in Equation (14). The AP of the 4th term $\Delta m_{3}$ is trivial because it is a (nontransformed) given process. Except for the 4th term, $\Delta m_{3}$ always appears with $\Delta m_{2}$ and $a$ as $\Delta m_{2}+a \Delta m_{3}$ with specific forms of $f^{2}(\Delta s)+a f^{3}(\Delta s)$, which satisfies the condition of a generalized second moment. Proposition 3.3 is therefore equivalent to a result under information set $x=\left(s, \tilde{m}_{2}\right)$ with a generalized second moment $\tilde{m}_{2}=m_{2}+a m_{3}$ that is obtained from $\tilde{f}^{2}=f^{2}+a f^{3}$. It implies that the additional information of $m_{3}$ to the information set does not produce any non-trivial function satisfying the AP. Related to this, Corollary 3.4 indicates that there is no realized fourth moment.

Corollary 3.4. When the information set is given by $x=\left(s, m_{2}, m_{3}\right)$, there is no realized 4 moment.

The proof is similar to that for Corollary 3.4.

\section{Arithmetic realized joint cumulants}

According to section 3, there is some skepticism about the geometric realized higher-order comoments. However, as mentioned in section 1 , financial studies state the importance of the higher-order comoments even above the fourth-order. Different from geometric comoments, arithmetic ones up to the fourth-order are available (recall Table 1). This section provides an investigation of the arithmetic comoments of general orders. Strictly speaking, our goal is to present realized joint cumulants. Because these are lesser-known, let us see their definitions.

Definition 4.1. Cumulants and joint cumulants

The $l$ th cumulant of a random variable $Y$ is defined by

$$
\kappa_{l}(Y)=\left.\frac{\partial^{l}}{\partial u^{l}} \ln E[\exp (u Y)]\right|_{u=0} .
$$


The joint cumulant of random variables $Y_{1}, Y_{2}, \ldots, Y_{l}$ is defined by

$$
\kappa\left(Y_{1}, Y_{2}, \ldots, Y_{l}\right)=\left.\frac{\partial^{l}}{\partial u_{1} \partial u_{2} \cdots \partial u_{l}} \ln E\left[\exp \left(\sum_{i=1}^{l} u_{i} Y_{i}\right)\right]\right|_{u_{1}=\cdots=u_{l}=0} .
$$

Recent studies such as Khademalomoom et al. (2019), Ahmed and Al Mafrachi (2021) and Cui et al. (2022) deal with the first six moments. Accordingly, the first six cumulants $\kappa_{l}(Y)$ are described in the second column of Table 2 . The cumulants are kinds of normalized moments because $\kappa_{l}(Y)=0$ for $l \geq 3$ when $Y$ follows a normal distribution. Moreover, a cumulant is a joint cumulant of an identical random variable with itself. In other words,

$$
\kappa_{l}(Y)=\kappa\left(Y_{1}, \ldots, Y_{l}\right), \quad \text { for } Y_{1}=\cdots=Y_{l}=Y .
$$

Moreover, for any constant number $a$, we have

$$
\kappa_{l}\left(Y_{M}+a Y\right)=\sum_{k=0}^{l} a^{k}\left(\begin{array}{l}
l \\
k
\end{array}\right) \kappa_{l-k, k}\left(Y_{M}, Y\right)
$$

where $\kappa_{l-k, k}\left(Y_{M}, Y\right)=\kappa\left(Y_{M}, \cdots, Y_{M}, Y, \cdots, Y\right)$ with $l-k Y_{M}$ s and $k Y_{\mathrm{S}}$, and $\kappa_{l-k, k}\left(Y_{M}, Y\right)$ is linked to the comoment $E\left[Y_{M}^{l-k} Y^{k}\right]$. For example, $\kappa_{0,1}\left(Y_{M}, Y\right), \ldots$ and $\kappa_{5,1}\left(Y_{M}, Y\right)$ are described in the third column of Table 2.

Fukasawa and Matsushita (2021) present the relationships between cumulants and the $\mathrm{AP}$, and the result is summarized as follows.

$$
\mathrm{E}_{0}\left[\sum_{j=1}^{N} B_{L}\left(X_{t_{j}}-X_{t_{j-1}}\right)\right]=E_{0}\left[B_{L}\left(X_{T}-X_{0}\right)\right]={ }^{0} \kappa_{L}\left(S_{T}\right),[5]
$$

where $B_{L}$ is the $L$ th complete Bell polynomial defined as

$$
B_{L}\left(y_{1}, \cdots, y_{L}\right)=\left.\frac{\partial^{L}}{\partial u^{L}} \exp \left(\sum_{l=1}^{L} \frac{u^{l}}{l !} y_{l}\right)\right|_{u=0},
$$

and $X_{t}=\left(S_{t}, M_{t}^{(2)}, M_{t}^{(3)}, \ldots, M_{t}^{(L-1)}, 0\right)$ with $M_{t}^{(l)}={ }^{t} \kappa_{l}\left(S_{T}\right)$. Equation (19) implies that

$$
\sum_{j=1}^{N} B_{L}\left(X_{t_{j}}-X_{t_{j-1}}\right)
$$

is an unbiased estimator of ${ }^{0} \kappa_{L}\left(S_{T}\right)$. Therefore, it can overcome drawbacks of conventional realized moments. Accordingly, the authors name Equation (21) the realized $L$ th cumulant. For illustration, the realized cumulants of orders 2-6 are presented in Table 3 . As stated in Neuberger (2012), Amaya et al. (2015), and Bae and Lee (2021), when $l$ is not two, each summand requires additional terms more than $\left(\Delta S_{t_{j}}\right)^{l}$. For example, $\Delta M_{t_{j}}^{(2)} \Delta S_{t_{j}}$ can reflect leverage effect when $l=3$, and $\Delta M_{t_{j}}^{(2)}\left(\Delta S_{t_{j}}\right)^{2}$ can reflect volatility structure when $l=4$.

By extending Fukasawa and Matsushita (2021), we provide realized joint cumulants in Proposition 4.2.

Proposition 4.2. For martingale processes $S_{1, t}$ and $S_{2, t}$, let us define $c M_{L-1,1}^{\text {real }}\left(S_{1}, S_{2}\right)$ as follows

$$
c M_{L-1,1}^{\text {real }}\left(S_{1}, S_{2}\right)=\sum_{j=1}^{N} \sum_{k=1}^{L-1}\left(\begin{array}{l}
L-1 \\
k
\end{array}\right) B_{L-k}\left(\Delta M_{t_{j}}^{(1,0)}, \cdots, \Delta M_{t_{j}}^{(L-k, 0)}\right) \Delta M_{t_{j}}^{(k-1,1)}
$$

Geometric and arithmetic comoments $-$ 7) 
JDQS

30,2

96

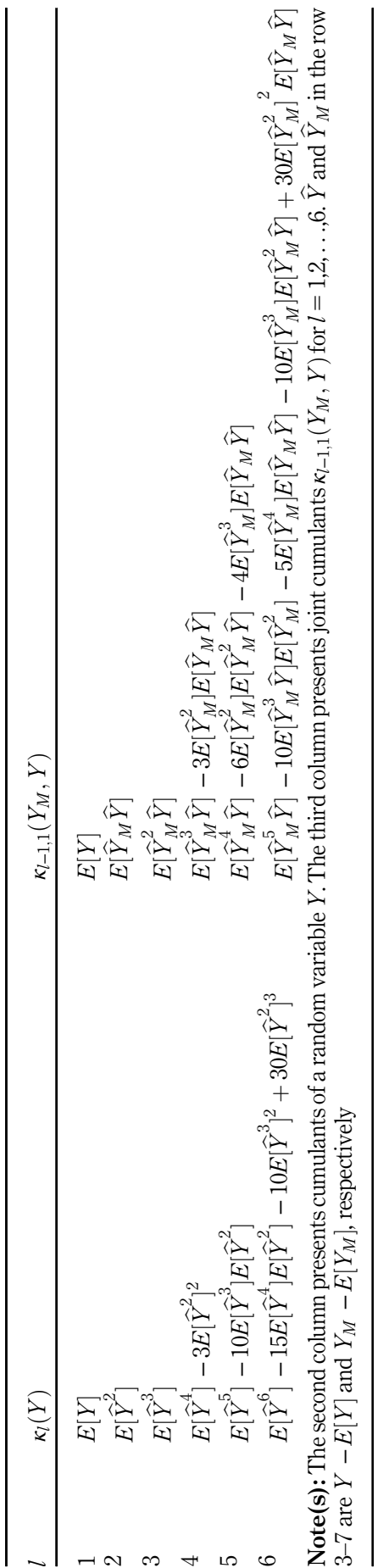

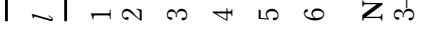

Table 2 .
The first six cumulants 


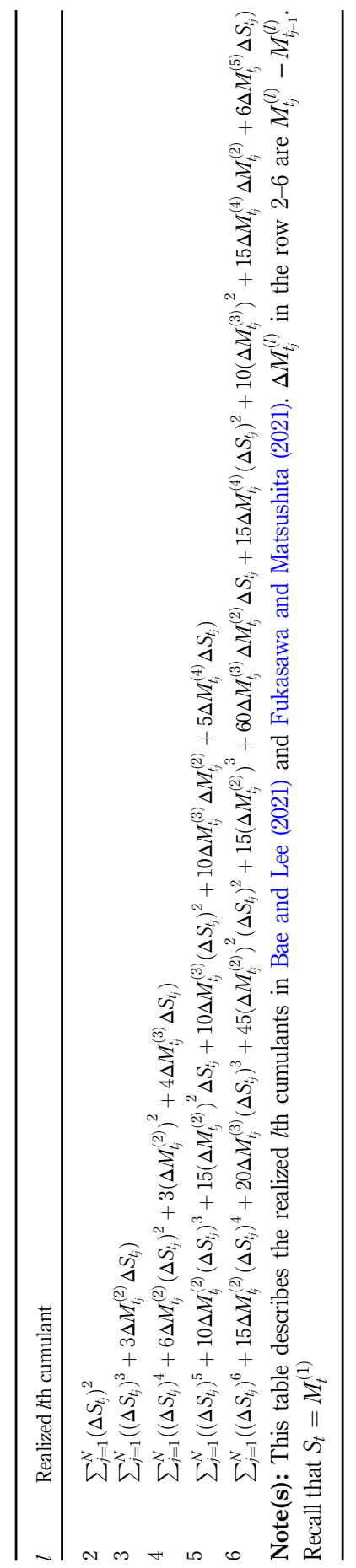

Geometric and arithmetic comoments

Table 3. Examples of realized cumulants 
JDQS

30,2

98

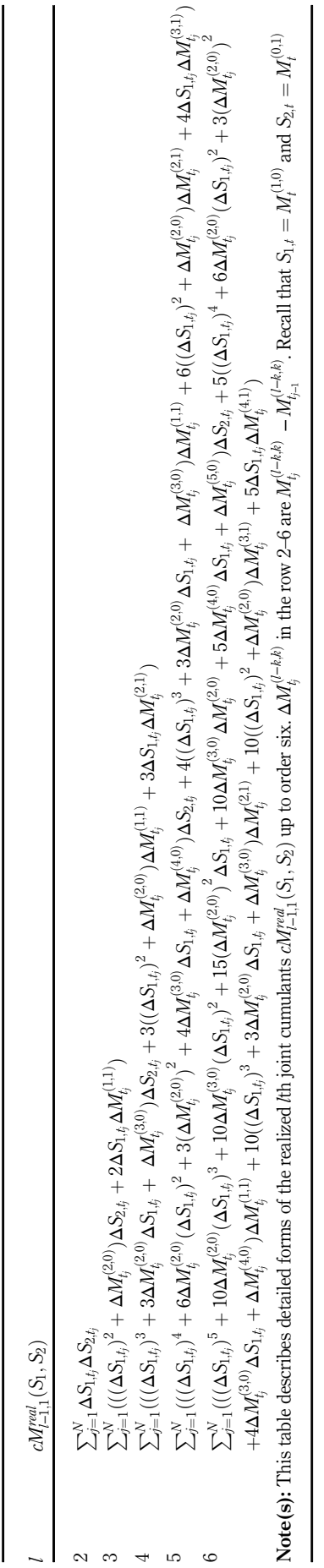

Table 4.

The 2-6 realized joint cumulants 
with $\Delta M_{t_{j}}^{(l-k, k)}=M_{t_{j}}^{(l-k, k)}-M_{t_{j-1}}^{(l-k, k)}$ and $M_{t}^{(l-k, k)}={ }^{t} \kappa_{l-k, k}\left(S_{1, T}, S_{2, T}\right)$. Then, we have

$$
E_{0}\left[c M_{L-1,1}^{\text {real }}\left(S_{1}, S_{2}\right)\right]={ }^{0} \kappa_{L-1,1}\left(S_{1, T}, S_{2, T}\right) .
$$

Proof is provided in Appendix 2.

Based on Proposition 4.2, we can measure the relationship between $S_{1}$ and $S_{2}$ well through $c M_{L-1,1}^{r e a l}\left(S_{1}, S_{2}\right)$. Because of Equation (23), it is an unbiased estimator of ${ }^{0} \kappa_{L-1,1}\left(S_{1, T}, S_{2, T}\right)$. Therefore, it can overcome drawbacks of conventional measures. For illustration, detailed forms of $c M_{l-1,1}^{\text {real }}\left(S_{1}, S_{2}\right)$ up to order six are presented in Table 4. Like the result of Table 3, it shows that the fifth joint cumulant $c M_{4,1}^{\text {real }}\left(S_{1}, S_{2}\right)$ requires more than $\sum_{j=1}^{N}\left(\Delta S_{1, t_{j}}\right)^{4} \Delta S_{2, t_{j}}$. For example, it additionally requires $\sum_{j=1}^{N} \Delta M_{t_{j}}^{(4,0)} \Delta S_{2, t_{j}}$, which is related to covariation between the second asset return and the kurtosis of the first asset return. Similarly, $c M_{5,1}^{\text {real }}\left(S_{1}, S_{2}\right)$ requires more than $\sum_{j=1}^{N}\left(\Delta S_{1, t_{j}}\right)^{5} \Delta S_{2, t_{j}}$

\section{Concluding remarks}

Neuberger (2012), Bae and Lee (2021), and Fukasawa and Matsushita (2021) demonstrate that realized third geometric moments and realized arithmetic moments of any orders are obtained by combining their lower-order implied moments and comoments. Extending the information set is therefore a natural trial to yield the higher order moments and comoments. Unlike previous studies, we show that geometric lower-order implied comoments do not yield geometric realized fourth moment and third comoment but yield geometric realized covariance only. The main reason for the non-existence is that the extension of the geometric information set does not produce additional non-trivial terms; the productions are only transformations of Neuberger (2012). Although this approach does not yield a meaningful measure, presenting this result can prevent the same trial and error for other scholars.

Furthermore, we yield the arithmetic realized lth joint cumulants, which are linked to $E\left[\left(S_{1, T}-S_{1,0}\right)^{l-1}\left(S_{2, T}-S_{2,0}\right)\right]$. Several financial theories apply them; for example, the extended CAPM includes $E\left[\left(r_{M}-E\left[r_{M}\right]\right)^{l-1}\left(r_{i}-E\left[r_{i}\right]\right)\right]$ for $l \geq 2$. Given the drawbacks of conventional realized comoments, we believe that empirical studies can use our measure in the future.

Depending on combinations of assets, there are other joint cumulants such as $E\left[\left(r_{M}-E\left[r_{M}\right]\right)^{l-2}\left(r_{i}-E\left[r_{i}\right]\right)^{2}\right]$ or $E\left[\left(r_{M}-E\left[r_{M}\right]\right)^{l-3}\left(r_{i}-E\left[r_{i}\right]\right)^{3}\right]$. We do not investigate them because they currently seem irrelevant to financial studies. However, we may obtain them as proof of Proposition 4.2 when the financial studies require them.

\section{Notes}

1. They use the realized moments for various purposes. Amaya et al. (2015) and Sim (2016) show that realized third moments can explain stock returns. Kim (2016) investigates the forecasting power of implied moments about realized moments. Mei et al. (2017) show realized third and fourth moments are related to future volatility. Kinateder and Papavassiliou (2019) show that realized fourth moment can predict sovereign bond returns during a crisis. Ahmed and Al Mafrachi (2021) show that realized moments up to the fifth-order can explain cryptocurrency returns.

2. Implied moments can be obtained from options (Bakshi and Madan, 2000; Bakshi et al., 2003; Kang et al., 2009; Neuberger, 2012).

3. Cumulants are normalized moments. See section 4 for details.

4. The rest of this section is preliminary of section 3 that proves the non-existence of the geometric realized comoments. Therefore, readers that are only interested in the form of the realized comoments should move to section 4 . 
JDQS 30,2
5. A left superscript $t$ of $\kappa$ means a time- $t$ conditional one. For example, ${ }^{t} \kappa_{L}(Y)=\left.\frac{\partial^{L}}{\partial u^{L}} \ln E_{t}[\exp (u Y)]\right|_{u=0}$.

6. The ten coefficients, $b_{0}, \cdots, b_{5}, b_{12}, b_{14}, b_{23}, b_{25}$, and $b_{26}$ are replaced with $d_{5}, \cdots, d_{14}$. More precisely, $\left(d_{5}, d_{8}, d_{12}, d_{13}\right)$ replace $\left(b_{1}, b_{4}, b_{12}, b_{25}\right),\left(d_{6}, d_{9}, d_{11}, d_{14}\right)$ replace $\left(b_{2}, b_{3}, b_{14}, b_{26}\right), d_{10}$ replaces $b_{23}$, and $d_{7}$ replaces $b_{0}$, given $b_{3}$ and $b_{12}$.

\section{References}

Ahmed, W.M. and Al Mafrachi, M. (2021), "Do higher-order realized moments matter for cryptocurrency returns?", International Review of Economics and Finance, Vol. 72, pp. 483-499.

Amaya, D., Christoffersen, P., Jacobs, K. and Vasquez, A. (2015), "Does realized skewness predict the cross-section of equity returns?", Journal of Financial Economics, Vol. 118, pp. 135-167.

Andersen, T.G., Bollerslev, T., Diebold, F.X. and Labys, P. (2003), "Modeling and forecasting realized volatility", Econometrica, Vol. 71, pp. 579-625.

Bae, K. and Lee, S. (2021), "Realized higher-order comoments", Quantitative Finance, Vol. 21, pp. 421-429.

Bakshi, G. and Madan, D. (2000), "Spanning and derivative-security valuation”, Journal of Financial Economics, Vol. 55, pp. 205-238.

Bakshi, G., Kapadia, N. and Madan, D. (2003), "Stock return characteristics, skew laws, and the differential pricing of individual equity options", Review of Financial Studies, Vol. 16, pp. 101-143.

Chung, Y.P., Johnson, H. and Schill, M.J. (2006), "Asset pricing when returns are nonnormal: Fama-French factors versus higher-order systematic comoments”, Journal of Business, Vol. 79, pp. 923-940.

Cui, J., Maghyereh, A., Goh, M. and Zou, H. (2022), "Risk spillovers and time-varying links between international oil and China's commodity futures markets: Fresh evidence from the higher-order moments", Energy, Vol. 238, 121751.

Dittmar, R.F. (2002), "Nonlinear pricing kernels, kurtosis preference, and evidence from the cross section of equity returns", Journal of Finance, Vol. 57, pp. 369-403.

Fukasawa, M. and Matsushita, K. (2021), "Realized cumulants for martingales", Electronic Communications in Probability, Vol. 26, pp. 1-10.

Hung, C.-H.D. (2008), "Momentum, size and value factors versus systematic co-moments in stock returns", Working paper, Durham University, UK.

Kang, B.J., Kang, S. and Yoon, S.-J. (2009), "Information content of adjusted implied volatility in the KOSPI 200 index options market”, Journal of Derivatives and Quantitative Studies, Vol. 17 No. 4, pp. $75-103$.

Khademalomoom, S., Narayan, P.K. and Sharma, S.S. (2019), "Higher moments and exchange rate behavior", Financial Review, Vol. 54, pp. 201-229.

Kim, S. (2016), "On the usefulness of risk-neutral skewness and kurtosis for forecasting the higher moments of stock returns", Journal of Derivatives and Quantitative Studies, Vol. 24 No. 2, pp. 185-220.

Kinateder, H. and Papavassiliou, V.G. (2019), "Sovereign bond return prediction with realized higher moments", Journal of International Financial Markets, Institutions and Money, Vol. 62, pp. 53-73.

Kraus, A. and Litzenberger, R.H. (1976), "Skewness preference and the valuation of risk assets", Journal of Finance, Vol. 31, pp. 1085-1100.

Mei, D., Liu, J., Ma, F. and Chen, W. (2017), "Forecasting stock market volatility: do realized skewness and kurtosis help?", Physica A: Statistical Mechanics and its Applications, Vol. 481, pp. 153-159.

Neuberger, A. (2012), "Realized skewness", Review of Financial Studies, Vol. 25, pp. 3423-3455.

Rubinstein, M.E. (1973), “A comparative statics analysis of risk premiums”, Journal of Business, Vol. 46, pp. 605-615.

Sim, M. (2016), "Realized skewness and the return predictability", Journal of Derivatives and Quantitative Studies, Vol. 24 No. 1, pp. 119-152. 


\section{Appendix 1}

Proofs for Propositions and Corollaries in Section 3

Beginnings of the proofs for Propositions 3.1 and 3.3 are identical. We denote them as common property A as follows:

Common property A: A common necessary condition of $g$ that satisfies the aggregation property.

Consider a vector-valued process $\left\{\left(\ln S_{1}(t), \ln S_{2}(t), M(t)\right): t=0,1,2\right\}$. In addition, let

$$
\begin{aligned}
& \left(\ln S_{1}, \ln S_{2}, M\right):(0,0, m) \rightarrow\left\{\begin{array}{lll}
\left(s_{1,1}, s_{2,1}, \alpha\right) & \rightarrow\left(s_{1,1}+\eta_{1}, s_{2,1}+\eta_{2}, \overrightarrow{0}\right) & \operatorname{Pr}=\pi_{1} \\
\left(s_{1,2}, s_{2,2}, \overrightarrow{0}\right) & \rightarrow\left(s_{1,2}, s_{2,2}, \overrightarrow{0}\right) & \operatorname{Pr}=\pi_{2} \\
\vdots & \vdots & \vdots \\
\left(s_{1, n}, s_{2, n}, \overrightarrow{0}\right) & \rightarrow\left(s_{1, n}, s_{2, n}, \overrightarrow{0}\right) & \operatorname{Pr}=\pi_{n}
\end{array}\right. \\
& \begin{array}{llllllll}
t: & 0 & \rightarrow & & 1 & \rightarrow & 2
\end{array}
\end{aligned}
$$

with $\sum_{j=1}^{n} \pi_{j}=1, \sum_{j=1}^{n} \pi_{j} \exp \left(s_{i, j}\right)=1, \mathrm{E}\left[\exp \left(\eta_{i}\right)\right]=1, \mathrm{E}\left[f^{k, l}\left(\eta_{1}, \eta_{2}\right)\right]=\alpha_{k, l}, \alpha=\left(\alpha_{2,0}, \alpha_{1,1}, \alpha_{0,2}, \alpha_{0,3}\right)$ and $m=\left(m_{2,0}, m_{1,1}, m_{0,2}, m_{0,3}\right)$ where

$$
m_{k, l}=\pi_{1} \mathrm{E}\left[f^{k, l}\left(s_{1,1}+\eta_{1}, s_{2,1}+\eta_{2}\right)\right]+\sum_{j=2}^{n} \pi_{j} f^{k, l}\left(s_{1, j}, s_{2, j}\right)
$$

and $f^{k, l}$ is a generalized moment function such that $f^{k, l}(0,0)=0, \lim _{(a, b) \rightarrow(0,0)} \frac{f^{k, l}(a, b)}{a^{k} b^{l}}=1, f^{k, l}(a, b)=f^{l, k}(b, a)$, and $f^{k}(a)=f^{k, 0}(a, b)$.

When the process satisfies the aggregation property, we have

$$
E\left[g\left(s_{1,1}+\eta_{1}, s_{2,1}+\eta_{2},-m\right)\right]=g\left(s_{1,1}, s_{2,1}, \alpha-m\right)+E\left[g\left(\eta_{1}, \eta_{2},-\alpha\right)\right]
$$

with $g(0, \cdots, 0)=0$. Differentiating Equation (A3) with respect to the $(k-2)$ th term of $m$, we obtain

$$
E\left[g_{k}\left(s_{1,1}+\eta_{1}, s_{2,1}+\eta_{2},-m\right)\right]=g_{k}\left(s_{1,1}, s_{2,1}, \alpha-m\right), \text { for } k=3, \cdots, 6,
$$

where $g_{k}$ is a partial differentiation with respect to the $k$ th term. By substituting $\left(s_{1,1}, s_{2,1}\right)=(0,0)$ and $m=\alpha$ into Equation (A4), we obtain:

$$
E\left[g_{k}\left(\eta_{1}, \eta_{2},-\alpha\right)\right]=g_{k}(0,0, \overrightarrow{0}), \text { for } k=3, \cdots, 6
$$

Then, by Lagrangian, we have

$$
\begin{aligned}
g_{k}\left(s_{1}, s_{2}, M\right)= & a_{k, 0}+A_{k, 1}(M)\left(e^{s_{1}}-1\right)+A_{k, 2}(M)\left(e^{s_{2}}-1\right)+A_{k, 3}(M)\left(f^{2}\left(s_{1}\right)+M_{2,0}\right) \\
& +A_{k, 4}(M)\left(f^{1,1}\left(\mathrm{~s}_{1}, s_{2}\right)+M_{1,1}\right)+A_{k, 5}(M)\left(f^{2}\left(s_{2}\right)+M_{0,2}\right) \\
& +A_{k, 6}(M)\left(f^{3}\left(s_{1}\right)+M_{3,0}\right)
\end{aligned}
$$

where $a_{k, 0}$ is a constant and $A_{k, 1}, \cdots, A_{k, 6}$ are functions of $M$. If we substitute Equation (A6), $\left(s_{1,1}, s_{2,1}\right)=(0,0)$ and $m=\alpha$ except for the $(l-2)$ th term into Equation (A4), we obtain: 
JDQS

30,2

$$
A_{k, l}(-m)\left(\alpha_{l-2}-m_{l-2}\right)=A_{k, l}\left(0, \cdots, 0, \alpha_{l-2}-m_{l-2}, 0, \cdots, 0\right)\left(\alpha_{l-2}-m_{l-2}\right) .
$$

Because $\pi, s_{i, j}$ and $\alpha$ are arbitrary, $A_{k, 3}(M), \cdots, A_{k, 6}(M)$ are constants. Thus, Equation (A7) is can be rewritten with notations of $a_{k, 3}, \ldots, a_{k, 6}$, as follows:

$$
\begin{aligned}
g_{k}\left(s_{1}, s_{2}, M\right)= & a_{k, 0}+A_{k, 1}(M)\left(e^{s_{1}}-1\right)+A_{k, 2}(M)\left(e^{s_{2}}-1\right)+a_{k, 3}\left(f^{2}\left(s_{1}\right)+M_{2,0}\right) \\
& +a_{k, 4}\left(f^{1,1}\left(\mathrm{~s}_{1}, s_{2}\right)+M_{1,1}\right)+a_{k, 5}\left(f^{2}\left(s_{2}\right)+M_{0,2}\right)+a_{k, 6}\left(f^{3}\left(s_{1}\right)+M_{3,0}\right) .
\end{aligned}
$$

To investigate $A_{k, 1}(M)$ and $A_{k, 2}(M)$, let us substitute (A8) into (A4) and differentiate it with respect to $m_{l}$. It yields

$$
\frac{\partial A_{k, 1}(-m)}{\partial m_{l}}=\frac{\partial A_{k, 1}(\alpha-m)}{\partial m_{l}}, \frac{\partial A_{k, 2}(-m)}{\partial m_{l}}=\frac{\partial A_{k, 2}(\alpha-m)}{\partial m_{l}} \text { for } l=3, \ldots, 6 .
$$

Therefore, $A_{k, 1}(M)$ and $A_{k, 2}(M)$ are affine functions. Accordingly, (A8) is represented as follows:

$$
\begin{aligned}
g_{k}\left(s_{1}, s_{2}, M\right)= & a_{k, 0}+\left(b_{k, 0}+b_{k, 1} M_{2,0}+b_{k, 2} M_{1,1}+b_{k, 3} M_{0,2}+b_{k, 4} M_{3,0}\right)\left(e^{s_{1}}-1\right) \\
& +\left(c_{k, 0}+c_{k, 1} M_{2,0}+c_{k, 2} M_{1,1}+c_{k, 3} M_{0,2}+c_{k, 4} M_{3,0}\right)\left(e^{s_{2}}-1\right) \\
& +a_{k, 3}\left(f^{2}\left(s_{1}\right)+M_{2,0}\right)+a_{k, 4}\left(f^{1,1}\left(\mathrm{~s}_{1}, s_{2}\right)+M_{1,1}\right)+a_{k, 5}\left(f^{2}\left(s_{2}\right)+M_{0,2}\right) \\
& +a_{k, 6}\left(f^{3}\left(s_{1}\right)+M_{3,0}\right) .
\end{aligned}
$$

\section{Proof for Proposition 3.1}

(Proof for the first statement)

We use the common property A with restricting the $M=\left(V_{1}, V_{2}, V_{c}\right)$ with $V_{1}=M_{2,0}, V_{2}=M_{0,2}$ and $V_{c}=M_{1,1}$. Similarly, we use notations $m=\left(v_{1}, v_{2}, v_{c}\right)$ and $\alpha=\left(\alpha_{1}, \alpha_{2}, \alpha_{c}\right)$. In addition, $f$ and $f_{c}$ replace $f^{2}$ and $f^{1,1}$, respectively. By integrating (A10) with respect to $V_{1}, V_{c}$ and $V_{2}$, we can obtain three different forms of $g\left(s_{1}, s_{2}, V_{1}, V_{2}, V_{c}\right)$ as follows.

$$
\begin{aligned}
g\left(s_{1}, s_{2}, V_{1}, V_{2}, V_{c}\right)= & a_{1,0} V_{1}+\left(b_{1,0} V_{1}+\frac{1}{2} b_{1,1} V_{1}^{2}+b_{1,2} V_{1} V_{c}+b_{1,3} V_{1} V_{2}\right)\left(e^{s_{1}}-1\right) \\
& +\left(c_{1,0} V_{1}+\frac{1}{2} c_{1,1} V_{1}^{2}+c_{1,2} V_{1} V_{c}+c_{1,3} V_{1} V_{2}\right)\left(e^{s_{2}}-1\right) \\
& +a_{1,3}\left(f\left(s_{1}\right) V_{1}+\frac{1}{2} V_{1}^{2}\right)+a_{1,4}\left(f_{c}\left(\mathrm{~s}_{1}, s_{2}\right) V_{1}+V_{1} V_{c}\right) \\
& +a_{1,5}\left(f\left(s_{2}\right) V_{1}+V_{1} V_{2}\right)+g^{1}\left(s_{1}, s_{2}, V_{2}, V_{c}\right) . \\
g\left(s_{1}, s_{2}, V_{1}, V_{2}, V_{c}\right)= & a_{2,0} V_{c}+\left(b_{2,0} V_{c}+b_{2,1} V_{1} V_{c}+\frac{1}{2} b_{2,2} V_{c}^{2}+b_{2,3} V_{c} V_{2}\right)\left(e^{s_{1}}-1\right) \\
& +\left(c_{2,0} V_{c}+c_{2,1} V_{1} V_{c}+\frac{1}{2} c_{2,2} V_{c}^{2}+c_{2,3} V_{c} V_{2}\right)\left(e^{s_{2}}-1\right) \\
& +a_{2,3}\left(f\left(s_{1}\right) V_{c}+V_{1} V_{c}\right)+a_{2,4}\left(f_{c}\left(\mathrm{~s}_{1}, s_{2}\right) V_{c}+\frac{1}{2} V_{c}^{2}\right) \\
& +a_{2,5}\left(f\left(s_{2}\right) V_{c}+V_{c} V_{2}\right)+g^{2}\left(s_{1}, s_{2}, V_{1}, V_{c}\right) .
\end{aligned}
$$




$$
\begin{aligned}
g\left(s_{1}, s_{2}, V_{1}, V_{2}, V_{c}\right)= & a_{3,0} V_{2}+\left(b_{3,0} V_{2}+b_{3,1} V_{1} V_{2}+b_{3,2} V_{c} V_{2}+\frac{1}{2} b_{3,3} V_{2}^{2}\right)\left(e^{s_{1}}-1\right) \\
& +\left(c_{3,0} V_{2}+c_{3,1} V_{1} V_{2}+c_{3,2} V_{c} V_{2}+\frac{1}{2} c_{3,3} V_{2}^{2}\right)\left(e^{s_{2}}-1\right) \\
& +a_{3,3}\left(f\left(s_{1}\right) V_{2}+V_{1} V_{2}\right)+a_{3,4}\left(f_{c}\left(\mathrm{~s}_{1}, s_{2}\right) V_{2}+V_{c} V_{2}\right) \\
& +a_{3,5}\left(f\left(s_{2}\right) V_{2}+\frac{1}{2} V_{2}^{2}\right)+g^{3}\left(s_{1}, s_{2}, V_{1}, V_{2}\right) .
\end{aligned}
$$

Geometric and arithmetic comoments

with some functions $g^{1}, g^{2}$, and $g^{3}$. By combining Equations (A11), (A12) and (A13), we obtain

$$
\begin{aligned}
g\left(s_{1}, s_{2}, V_{1}, V_{2}, V_{c}\right)= & b_{0} V_{c}+b_{1} V_{1}+b_{2} V_{2}+\left(e^{s_{1}}-1\right)\left(b_{3} V_{c}+b_{4} V_{1}+b_{5} V_{2}\right. \\
& \left.+b_{6} V_{c} V_{1}+b_{7} V_{c} V_{2}+b_{8} V_{1} V_{2}+b_{9} V_{c}^{2}+b_{10} V_{1}^{2}+b_{11} V_{2}^{2}\right) \\
& +\left(e^{s_{2}}-1\right)\left(b_{12} V_{c}+b_{13} V_{1}+b_{14} V_{2}+b_{15} V_{c} V_{1}\right. \\
& \left.+b_{16} V_{c} V_{2}+b_{17} V_{1} V_{2}+b_{18} V_{c}^{2}+b_{19} V_{1}^{2}+b_{20} V_{2}^{2}\right) \\
& +b_{21}\left(f\left(s_{1}\right) V_{c}+V_{1} V_{c}+V_{1} f_{c}\left(s_{1}, s_{2}\right)\right) \\
& +b_{22}\left(f\left(s_{2}\right) V_{c}+V_{2} V_{c}+V_{2} f_{c}\left(s_{1}, s_{2}\right)\right) \\
& +b_{23}\left(f\left(s_{2}\right) V_{1}+V_{1} V_{2}+f\left(s_{1}\right) V_{2}\right)+b_{24}\left(2 f_{c}\left(s_{1}, s_{2}\right)+V_{c}\right) V_{c} \\
& +b_{25}\left(2 f\left(s_{1}\right)+V_{1}\right) V_{1}+b_{26}\left(2 f\left(s_{2}\right)+V_{2}\right) V_{2}+g^{s}\left(s_{1}, s_{2}\right)
\end{aligned}
$$

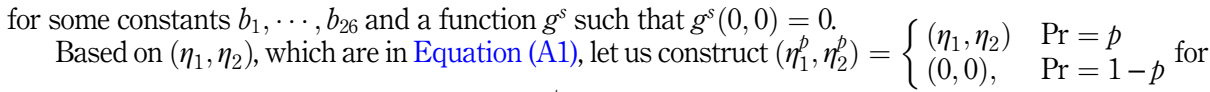
a constant $p$ in $[0,1]$. For $i \in\{1,2\}$, we have $E\left[e^{\eta_{i}^{p}}\right]=1, E\left[f\left(\eta_{i}^{p}\right)\right]=\alpha_{i} p$ and $E\left[f_{c}\left(\eta_{1}^{p}, \eta_{2}^{p}\right)\right]=\alpha_{c} p$. Then, by substituting Equation (A14) into (A3) and $\left(\eta_{1}^{p}, \eta_{2}^{p}\right)$ into $\left(\eta_{1}, \eta_{2}\right)$, we obtain:

$$
\begin{aligned}
& 0=p\left(e^{s_{11}}-1\right)\left(\begin{array}{l}
b_{3} \alpha_{c}+b_{4} \alpha_{1}+b_{5} \alpha_{2}+b_{6}\left(\alpha_{c} \alpha_{1} p-\alpha_{1} v_{c}-\alpha_{c} v_{1}\right) \\
+b_{7}\left(\alpha_{2} \alpha_{c} p-\alpha_{2} v_{c}-\alpha_{c} v_{2}\right)+b_{8}\left(\alpha_{1} \alpha_{2} p-\alpha_{2} v_{1}-\alpha_{1} v_{2}\right) \\
+b_{9}\left(\alpha_{c}^{2} p-2 \alpha_{c} v_{c}\right)+b_{10}\left(\alpha_{1}^{2} p-2 \alpha_{1} v_{1}\right)+b_{11}\left(\alpha_{2}^{2} p-2 \alpha_{2} v_{2}\right)
\end{array}\right) \\
& +p\left(e^{s_{21}}-1\right)\left(\begin{array}{l}
b_{12} \alpha_{c}+b_{13} \alpha_{1}+b_{14} \alpha_{2}+b_{15}\left(\alpha_{c} \alpha_{1} p-\alpha_{1} v_{c}-\alpha_{c} v_{1}\right) \\
+b_{16}\left(\alpha_{2} \alpha_{c} p-\alpha_{2} v_{c}-\alpha_{c} v_{2}\right)+b_{17}\left(\alpha_{1} \alpha_{2} p-\alpha_{2} v_{1}-\alpha_{1} v_{2}\right) \\
+b_{18}\left(\alpha_{c}^{2} p-2 \alpha_{c} v_{c}\right)+b_{19}\left(\alpha_{1}^{2} p-2 \alpha_{1} v_{1}\right)+b_{20}\left(\alpha_{2}^{2} p-2 \alpha_{2} v_{2}\right)
\end{array}\right) \\
& +p b_{21}\left(\begin{array}{l}
\left(E\left[f\left(s_{11}+\eta_{1}\right)\right]-f\left(s_{11}\right)-\alpha_{1}\right) v_{c}+f\left(s_{11}\right) \alpha_{c}+\alpha_{1} f_{c}\left(s_{11}, s_{21}\right) \\
+\left(E\left[f_{c}\left(s_{11}+\eta_{1}, s_{21}+\eta_{2}\right)\right]-f_{c}\left(s_{11}, s_{21}\right)-\alpha_{c}\right) v_{1}
\end{array}\right) \\
& +p b_{22}\left(\begin{array}{l}
\left(E\left[f\left(s_{21}+\eta_{2}\right)\right]-f\left(s_{21}\right)-\alpha_{2}\right) v_{c}+f\left(s_{21}\right) \alpha_{c}+\alpha_{2} f_{c}\left(s_{11}, s_{21}\right) \\
+\left(E\left[f_{c}\left(s_{11}+\eta_{1}, s_{21}+\eta_{2}\right)\right]-f_{c}\left(s_{11}, s_{21}\right)-\alpha_{c}\right) v_{2}
\end{array}\right) \\
& +p b_{23}\left(\begin{array}{l}
\left(E\left[f\left(s_{21}+\eta_{2}\right)\right]-f\left(s_{21}\right)-\alpha_{2}\right) v_{1}+f\left(s_{21}\right) \alpha_{1}+f\left(s_{11}\right) \alpha_{2} \\
+\left(E\left[f\left(s_{11}+\eta_{1}\right)\right]-f\left(s_{11}\right)-\alpha_{1}\right) v_{2}
\end{array}\right) \\
& +2 p b_{24}\left(\left(E\left[f_{c}\left(s_{11}+\eta_{1}, s_{21}+\eta_{2}\right)\right]-f_{c}\left(s_{11}, s_{21}\right)-\alpha_{c}\right) v_{c}+f_{c}\left(s_{11}, s_{21}\right) \alpha_{c}\right) \\
& +2 p b_{25}\left(\left(E\left[f\left(s_{11}+\eta_{1}\right)\right]-f\left(s_{11}\right)-\alpha_{1}\right) v_{1}+f\left(s_{11}\right) \alpha_{1}\right) \\
& +2 p b_{26}\left(\left(E\left[f\left(s_{21}+\eta_{2}\right)\right]-f\left(s_{21}\right)-\alpha_{2}\right) v_{2}+f\left(s_{21}\right) \alpha_{2}\right) \\
& -p E\left[g^{s}\left(s_{11}+\eta_{1}, s_{21}+\eta_{2}\right)\right]+p g^{s}\left(s_{1}, s_{2}\right)+p E\left[g^{s}\left(\eta_{1}, \eta_{2}\right)\right]
\end{aligned}
$$


JDQS

30,2

\section{4}

Because (A15) holds for arbitrary $p$, the coefficient of $p^{2}$ should be zero.

$$
\begin{aligned}
0= & \left(e^{s_{11}}-1\right)\left(b_{6} \alpha_{c} \alpha_{1}+b_{7} \alpha_{2} \alpha_{c}+b_{8} \alpha_{1} \alpha_{2}+b_{9} \alpha_{c}^{2}+b_{10} \alpha_{1}^{2}+b_{11} \alpha_{2}^{2}\right) \\
& +\left(e^{s_{21}}-1\right)\left(b_{15} \alpha_{c} \alpha_{1}+b_{16} \alpha_{2} \alpha_{c}+b_{17} \alpha_{1} \alpha_{2}+b_{18} \alpha_{c}^{2}+b_{19} \alpha_{1}^{2}+b_{20} \alpha_{2}^{2}\right)
\end{aligned}
$$

Furthermore, because $s_{11}$ and $s_{21}$ are arbitrary, we have:

$$
\begin{gathered}
b_{6} \alpha_{c} \alpha_{1}+b_{7} \alpha_{2} \alpha_{c}+b_{8} \alpha_{1} \alpha_{2}+b_{9} \alpha_{c}^{2}+b_{10} \alpha_{1}^{2}+b_{11} \alpha_{2}^{2}=0 \\
b_{15} \alpha_{c} \alpha_{1}+b_{16} \alpha_{2} \alpha_{c}+b_{17} \alpha_{1} \alpha_{2}+b_{18} \alpha_{c}^{2}+b_{19} \alpha_{1}^{2}+b_{20} \alpha_{2}^{2}=0
\end{gathered}
$$

Given $\alpha_{1}$ and $\alpha_{2}$, we can construct arbitrary $\alpha_{c}$. Therefore, Equation (A17) yields:

$$
b_{9}=b_{6} \alpha_{1}+b_{7} \alpha_{2}=b_{8} \alpha_{1} \alpha_{2}+b_{10} \alpha_{1}^{2}+b_{11} \alpha_{2}^{2}=0
$$

Adopting this logic to Equation (A18) instead of (A17) and to $\alpha_{1}$ or $\alpha_{2}$ instead of $\alpha_{c}$, we can obtain

$$
b_{6}=b_{7}=\cdots=b_{11}=0 \text { and } b_{15}=b_{16}=\cdots=b_{20}=0
$$

Additionally, because the coefficient of $p$ in Equation (A15) is zero, we have:

$$
\begin{aligned}
0 & =\left(e^{s_{11}}-1\right)\left(b_{3} \alpha_{c}+b_{4} \alpha_{1}+b_{5} \alpha_{2}\right)+\left(e^{s_{21}}-1\right)\left(b_{12} \alpha_{c}+b_{13} \alpha_{1}+b_{14} \alpha_{2}\right) \\
& +b_{21}\left(\begin{array}{l}
\left(E\left[f\left(s_{11}+\eta_{1}\right)\right]-f\left(s_{11}\right)-\alpha_{1}\right) v_{c}+f\left(s_{11}\right) \alpha_{c}+\alpha_{1} f_{c}\left(s_{11}, s_{21}\right) \\
+\left(E\left[f_{c}\left(s_{11}+\eta_{1}, s_{21}+\eta_{2}\right)\right]-f_{c}\left(s_{11}, s_{21}\right)-\alpha_{c}\right) v_{1}
\end{array}\right) \\
& +b_{22}\left(\begin{array}{l}
\left(E\left[f\left(s_{21}+\eta_{2}\right)\right]-f\left(s_{21}\right)-\alpha_{2}\right) v_{c}+f\left(s_{21}\right) \alpha_{c}+\alpha_{2} f_{c}\left(s_{11}, s_{21}\right) \\
+\left(E\left[f_{c}\left(s_{11}+\eta_{1}, s_{21}+\eta_{2}\right)\right]-f_{c}\left(s_{11}, s_{21}\right)-\alpha_{c}\right) v_{2}
\end{array}\right) \\
& +b_{23}\left(\begin{array}{l}
\left(E\left[f\left(s_{21}+\eta_{2}\right)\right]-f\left(s_{21}\right)-\alpha_{2}\right) v_{1}+f\left(s_{21}\right) \alpha_{1}+f\left(s_{11}\right) \alpha_{2} \\
+\left(E\left[f\left(s_{11}+\eta_{1}\right)\right]-f\left(s_{11}\right)-\alpha_{1}\right) v_{2}
\end{array}\right) \\
& +2 b_{24}\left(\left(E\left[f_{c}\left(s_{11}+\eta_{1}, s_{21}+\eta_{2}\right)\right]-f_{c}\left(s_{11}, s_{21}\right)-\alpha_{c}\right) v_{c}+f_{c}\left(s_{11}, s_{21}\right) \alpha_{c}\right) \\
& +2 b_{25}\left(\left(E\left[f\left(s_{11}+\eta_{1}\right)\right]-f\left(s_{11}\right)-\alpha_{1}\right) v_{1}+f\left(s_{11}\right) \alpha_{1}\right) \\
& +2 b_{26}\left(\left(E\left[f\left(s_{21}+\eta_{2}\right)\right]-f\left(s_{21}\right)-\alpha_{2}\right) v_{2}+f\left(s_{21}\right) \alpha_{2}\right) \\
& -E\left[g^{s}\left(s_{11}+\eta_{1}, s_{21}+\eta_{2}\right)\right]+g^{s}\left(s_{1}, s_{2}\right)+E\left[g^{s}\left(\eta_{1}, \eta_{2}\right)\right]
\end{aligned}
$$

Because $v_{c}$ is arbitrary, the coefficient of $v_{c}$ is zero. Thus, we have

$$
\begin{aligned}
& b_{21}\left(E\left[f\left(s_{11}+\eta_{1}\right)\right]-f\left(s_{11}\right)-\alpha_{1}\right)+b_{22}\left(E\left[f\left(s_{21}+\eta_{2}\right)\right]-f\left(s_{21}\right)-\alpha_{2}\right) \\
& \quad+2 b_{24}\left(E\left[f_{c}\left(s_{11}+\eta_{1}, s_{21}+\eta_{2}\right)\right]-f_{c}\left(s_{11}, s_{21}\right)-\alpha_{c}\right)=0
\end{aligned}
$$

Now, consider a random variable $\eta_{3}$ with $\eta_{3} \stackrel{d}{=} \eta_{2}$ and $E\left[f_{c}\left(\eta_{1}, \eta_{3}\right)\right] \neq E\left[f_{c}\left(\eta_{1}, \eta_{2}\right)\right]$. Then,

$$
\begin{gathered}
b_{21}\left(E\left[f\left(s_{11}+\eta_{1}\right)\right]-f\left(s_{11}\right)-\alpha_{1}\right)+b_{22}\left(E\left[f\left(s_{21}+\eta_{3}\right)\right]-f\left(s_{21}\right)-\alpha_{2}\right) \\
\quad+2 b_{24}\left(E\left[f_{c}\left(s_{11}+\eta_{1}, s_{21}+\eta_{3}\right)\right]-f_{c}\left(s_{11}, s_{21}\right)-E\left[f_{c}\left(\eta_{1}, \eta_{3}\right)\right]\right)=0
\end{gathered}
$$

By subtracting Equation (A23) from Equation (A22), one can see that $b_{24}=0$ or

$$
E\left[f_{c}\left(s_{11}+\eta_{1}, s_{21}+\eta_{2}\right)\right]=E\left[f_{c}\left(s_{11}+\eta_{1}, s_{21}+\eta_{3}\right)\right]+E\left[f_{c}\left(\eta_{1}, \eta_{2}\right)\right]-E\left[f_{c}\left(\eta_{1}, \eta_{3}\right)\right] .
$$

When we substitute

$$
\left(\eta_{1}, \eta_{2}, \eta_{3}\right)= \begin{cases}(\ln (1+k), \ln (1+k), \ln (1-k)) & \operatorname{Pr}=1 / 2 \\ (\ln (1-k), \ln (1-k), \ln (1+k)) & \operatorname{Pr}=1 / 2\end{cases}
$$


into Equation (A24) and multiply both sides of the equation with $\frac{1}{2 k^{2}}$, and take the limit with $k \rightarrow 0$, we get

$$
\frac{\partial^{2} f_{c}(x, y)}{\partial x \partial y}=1
$$

Hence,

$$
f_{c}\left(s_{11}, s_{21}\right)=s_{11} s_{21}+F_{1}\left(s_{11}\right)+F_{2}\left(s_{21}\right)
$$

for some functions $F_{1}$ and $F_{2}$. Then, applying the condition of $\lim _{(x, y) \rightarrow(0,0)} \frac{f_{c}(x, y)}{x y}=1$, we obtain $f_{c}\left(s_{11}, s_{21}\right)=s_{11} s_{21}$. By substituting it into Equation (A22), we obtain the following equation:

$$
\begin{aligned}
& b_{21}\left(E\left[f\left(s_{11}+\eta_{1}\right)\right]-f\left(s_{11}\right)-\alpha_{1}\right)+b_{22}\left(E\left[f\left(s_{21}+\eta_{2}\right)\right]-f\left(s_{21}\right)-\alpha_{2}\right)+2 b_{24}\left(s_{11} E\left[\eta_{2}\right]\right. \\
& \left.\quad+s_{21} E\left[\eta_{1}\right]\right)=0
\end{aligned}
$$

When $s_{11}=0$, Equation (A28) becomes $b_{22}\left(E\left[f\left(s_{21}+\eta_{2}\right)\right]-f\left(s_{21}\right)-\alpha_{2}\right)+2 b_{24} s_{21} E\left[\eta_{1}\right]=0$. Because $\eta_{1}$ can be chosen independently on $s_{21}$ and $\eta_{2}$,

$$
b_{24}=0 .
$$

The logic between Equations (A22) and (A29) shows that multiplier of $E\left[f_{c}\left(s_{11}+\eta_{1}\right.\right.$, $\left.\left.s_{21}+\eta_{2}\right)\right]-f_{c}\left(s_{11}, s_{21}\right)-\alpha_{c}$ is zero. For alternatives of Equation (A22), as the coefficients of $v_{1}$ and $v_{2}$ instead of $v_{c}$ in Equation (A21), the same logic yields

$$
b_{21}=b_{22}=0 .
$$

Because the coefficients of $v_{1}$ and $v_{2}$ in Equation (A21) are 0, equations (A29) and (A30) implies:

$$
\begin{aligned}
& b_{23}\left(E\left[f\left(s_{21}+\eta_{2}\right)\right]-f\left(s_{21}\right)-\alpha_{2}\right)+2 b_{25}\left(E\left[f\left(s_{11}+\eta_{1}\right)\right]-f\left(s_{11}\right)-\alpha_{1}\right) \\
& =b_{23}\left(E\left[f\left(s_{11}+\eta_{1}\right)\right]-f\left(s_{11}\right)-\alpha_{1}\right)+2 b_{26}\left(E\left[f\left(s_{21}+\eta_{2}\right)\right]-f\left(s_{21}\right)-\alpha_{2}\right)=0 .
\end{aligned}
$$

Substituting $s_{11}=0$ or $s_{21}=0$ into the (A31) yields:

$$
\begin{aligned}
& b_{23}\left(E\left[f\left(s_{21}+\eta_{2}\right)\right]-f\left(s_{21}\right)-\alpha_{2}\right)=b_{25}\left(E\left[f\left(s_{11}+\eta_{1}\right)\right]-f\left(s_{11}\right)-\alpha_{1}\right) \\
& =b_{23}\left(E\left[f\left(s_{11}+\eta_{1}\right)\right]-f\left(s_{11}\right)-\alpha_{1}\right)=b_{26}\left(E\left[f\left(s_{21}+\eta_{2}\right)\right]-f\left(s_{21}\right)-\alpha_{2}\right)=0
\end{aligned}
$$

Thus,

$$
E[f(s+\eta)]-f(s)-E[f(\eta)]=0 \text { or } b_{23}=b_{25}=b_{26}=0 .
$$

Here, according to Neuberger (2012), $E[f(s+\eta)]-f(s)-E[f(\eta)]=0$ is equivalent to

$$
f(x)=2\left(e^{x}-1-x\right) .
$$

In sum, combining (A21) with (A29), (A30) and (A33) yields

$$
\begin{aligned}
0= & \left(e^{s_{11}}-1\right)\left(b_{3} \alpha_{c}+b_{4} \alpha_{1}+b_{5} \alpha_{2}\right)+\left(e^{s_{21}}-1\right)\left(b_{12} \alpha_{c}+b_{13} \alpha_{1}+b_{14} \alpha_{2}\right) \\
& +b_{23}\left(f\left(s_{21}\right) \alpha_{1}+f\left(s_{11}\right) \alpha_{2}\right)+2 b_{25} f\left(s_{11}\right) \alpha_{1}+2 b_{26} f\left(s_{21}\right) \alpha_{2} \\
& -E\left[g^{s}\left(s_{11}+\eta_{1}, s_{21}+\eta_{2}\right)\right]+g^{s}\left(s_{1}, s_{2}\right)+E\left[g^{s}\left(\eta_{1}, \eta_{2}\right)\right]
\end{aligned}
$$
To Equation (A35), multiplying $2 / k^{2}$, substituting $\left(\eta_{1}, \eta_{2}\right)=\left\{\begin{array}{ll}(\ln (1+k), 0) & \operatorname{Pr}=1 / 2 \\ (\ln (1-k), 0) & \operatorname{Pr}=1 / 2\end{array}\right.$, and taking
the limit yields: 
JDQS

30,2

106

$$
\begin{aligned}
0= & 2 b_{4}\left(e^{s_{11}}-1\right)+2 b_{13}\left(e^{s_{21}}-1\right)+4 b_{23}\left(e^{s_{21}}-1-s_{21}\right)+8 b_{25}\left(e^{s_{11}}-1-s_{11}\right) \\
& -\frac{\partial^{2} g^{s}\left(s_{11}, s_{21}\right)}{\partial s_{11}^{2}}+\frac{\partial g^{s}\left(s_{11}, s_{21}\right)}{\partial s_{11}}+\frac{\partial^{2} g^{s}(0,0)}{\partial s_{11}^{2}}-\frac{\partial g^{s}(0,0)}{\partial s_{11}} .
\end{aligned}
$$

Similarly, when use $\left(\eta_{1}, \eta_{2}\right)=\left\{\begin{array}{ll}(0, \ln (1+k)) & \operatorname{Pr}=1 / 2 \\ (0, \ln (1-k)) & \operatorname{Pr}=1 / 2\end{array}\right.$, we can obtain

$$
\begin{aligned}
0= & 2 b_{5}\left(e^{s_{11}}-1\right)+2 b_{14}\left(e^{s_{21}}-1\right)+4 b_{23}\left(e^{s_{11}}-1-s_{11}\right)+8 b_{26}\left(e^{s_{21}}-1-s_{21}\right) \\
& -\frac{\partial^{2} g^{s}\left(s_{11}, s_{21}\right)}{\partial s_{21}^{2}}+\frac{\partial g^{s}\left(s_{11}, s_{21}\right)}{\partial s_{21}}+\frac{\partial^{2} g^{s}(0,0)}{\partial s_{21}^{2}}-\frac{\partial g^{s}(0,0)}{\partial s_{21}} .
\end{aligned}
$$

Alternatively, let us multiply $\frac{1}{2 k^{2}}$ to Equation (A35), substitute (A38) and (A39) into Equation (A35), and subtract the equation obtained by the former substitution from that obtained by the latter; then, by taking limits, we get (A40).

$$
\begin{array}{r}
\left(\eta_{1}, \eta_{2}\right)= \begin{cases}(\ln (1+k), \ln (1+k)) & \mathrm{Pr}=1 / 2 \\
(\ln (1-k), \ln (1-k)) & \mathrm{Pr}=1 / 2\end{cases} \\
\left(\eta_{1}, \eta_{2}\right)= \begin{cases}(\ln (1+k), \ln (1-k)) & \mathrm{Pr}=1 / 2 \\
(\ln (1-k), \ln (1+k)) & \mathrm{Pr}=1 / 2\end{cases} \\
0=b_{3}\left(e^{s_{11}}-1\right)+b_{12}\left(e^{s_{21}}-1\right)-\frac{\partial^{2} g^{s}\left(s_{11}, s_{21}\right)}{\partial s_{11} s_{21}}+\frac{\partial^{2} g^{s}(0,0)}{\partial s_{11} s_{21}}
\end{array}
$$

Then, the solutions of Equation (A40), (A36) and (A37) are given as

$$
\begin{aligned}
g^{s}(x, y)= & b_{3}\left(e^{x}-x\right) y+b_{12}\left(e^{y}-y\right) x+h_{1}(x)+h_{2}(y)+b_{27} x y \\
g^{s}(x, y)= & 2 b_{4}\left(e^{x} x-e^{x}+x\right)-2 b_{13}\left(e^{y}-1\right) x-4 b_{23} x\left(e^{y}-y-1\right) \\
& +4 b_{25}\left(2 e^{x} x-2 e^{x}+x^{2}+4 x\right)+e^{x} h_{3}(y)+h_{4}(y)+b_{28} x \\
g^{s}(x, y)= & 2 b_{14}\left(e^{y} y-e^{y}+y\right)-2 b_{5}\left(e^{x}-1\right) y-4 b_{23} y\left(e^{x}-x-1\right) \\
& +4 b_{26}\left(2 e^{y} y-2 e^{y}+y^{2}+4 y\right)+e^{y} h_{5}(x)+h_{6}(x)+b_{29} y
\end{aligned}
$$

for some functions $h_{1}, \cdots, h_{6}$ and constants $b_{i}$. Therefore, $g^{s}(x, y)$ is a linear combination of $e^{x} y, e^{y} x, x y$, $e^{x} x, e^{x}, x^{2}, x, e^{y} y, e^{y}, y^{2}, y$ and 1 . The consistency in the coefficients of $e^{x} y$ and $e^{y} x$ requires $b_{5}=-\frac{1}{2} b_{3}-2 b_{23}$ and $b_{13}=-\frac{1}{2} b_{12}-2 b_{23}$. Thus, by Equation (A14), (A20), (A29), (A30), (A34), (A41), (A42), (A43) and $g^{s}(0,0)=0, g$ and $g^{s}$ are given by

$$
\begin{aligned}
& g\left(s_{1}, s_{2}, V_{1}, V_{2}, V_{c}\right)=b_{0} V_{c}+b_{1} V_{1}+b_{2} V_{2} \\
& +\left(b_{3} V_{c}+b_{4} V_{1}-\left(\frac{1}{2} b_{3}+2 b_{23}\right) V_{2}\right)\left(e^{s_{1}}-1\right) \\
& \quad+\left(b_{12} V_{c}-\left(\frac{1}{2} b_{12}+2 b_{23}\right) V_{1}+b_{14} V_{2}\right)\left(e^{s_{2}}-1\right) \\
& +b_{23}\left(2\left(e^{s_{2}}-s_{2}-1\right) V_{1}+V_{1} V_{2}+2\left(e^{s_{1}}-s_{1}-1\right) V_{2}\right) \\
& +b_{25}\left(4\left(e^{s_{1}}-s_{1}-1\right)+V_{1}\right) V_{1}+b_{26}\left(4\left(e^{s_{2}}-s_{2}-1\right)+V_{2}\right) V_{2}+g^{s}\left(s_{1}, s_{2}\right), \\
& g^{s}\left(s_{1}, s_{2}\right)=d_{1}\left(e^{s_{1}}-1\right)+d_{2} s_{1}+d_{3}\left(e^{s_{2}}-1\right)+d_{4} s_{2}+4 b_{23} s_{1} s_{2}+4 b_{25} s_{1}^{2} \\
& \quad+4 b_{26} s_{2}^{2}+b_{3} e^{s_{1}} s_{2}+b_{12} e^{s_{2}} s_{1}+\left(2 b_{4}+8 b_{25}\right) e^{s_{1}} s_{1}+\left(2 b_{14}+8 b_{26}\right) e^{s_{2}} s_{2} .
\end{aligned}
$$


(A44) and (A45) can be arranged as

$$
\begin{aligned}
g\left(s_{1}, s_{2}, V_{1}, V_{2}, V_{c}\right)= & d_{1}\left(e^{s_{1}}-1\right)+d_{2} s_{1}+d_{3}\left(e^{s_{2}}-1\right)+d_{4} s_{2}+d_{5} V_{1}+d_{6} V_{2}+d_{7} V_{c} \\
& +d_{8}\left(V_{1}-2 s_{1}\right)^{2}+d_{9}\left(V_{2}-2 s_{2}\right)^{2}+d_{10}\left(V_{1}-2 s_{1}\right)\left(V_{2}-2 s_{2}\right) \\
& +d_{11} e^{s_{1}}\left(2 V_{c}-V_{2}+2 s_{2}\right)+d_{12} e^{s_{2}}\left(2 V_{c}-V_{1}+2 s_{1}\right) \\
& +d_{13} e^{s_{1}}\left(V_{1}+2 s_{1}\right)+d_{14} e^{s_{2}}\left(V_{2}+2 s_{2}\right)
\end{aligned}
$$

Geometric and arithmetic comoments

(A46)

where $d_{5}=b_{1}-b_{4}+\frac{1}{2} b_{12}-4 b_{25}, d_{6}=b_{2}+\frac{1}{2} b_{3}-b_{14}-4 b_{26}, d_{7}=b_{0}-b_{3}-b_{12}, d_{8}=b_{25}, d_{9}=b_{26}$, $d_{10}=b_{23}, d_{11}=\frac{1}{2} b_{3}, d_{12}=\frac{1}{2} b_{12}, d_{13}=b_{4}+4 b_{25}$ and $d_{14}=b_{14}+4 b_{26}[6]$.

Substituting these into equation (A3) yields the following:

$$
\begin{aligned}
0 & =2 d_{8}\left(-v_{1}-2 s_{11}+\alpha_{1}\right)\left(\alpha_{1}+2 E\left[\eta_{1}\right]\right)+2 d_{9}\left(-v_{2}-2 s_{21}+\alpha_{2}\right)\left(\alpha_{2}+2 E\left[\eta_{2}\right]\right) \\
& +d_{10}\left(\left(-v_{1}-2 s_{11}+\alpha_{1}\right)\left(\alpha_{2}+2 E\left[\eta_{2}\right]\right)+\left(-v_{2}-2 s_{21}+\alpha_{2}\right)\left(\alpha_{1}+2 E\left[\eta_{1}\right]\right)\right) \\
& +\left(e^{s_{11}}-1\right)\left(d_{13}\left(\alpha_{1}-2 E\left[\eta_{1} e^{\eta_{1}}\right]\right)+d_{11}\left(2 \alpha_{c}-2 E\left[\eta_{2} e^{\eta_{1}}\right]-\alpha_{2}\right)\right) \\
& +\left(e^{s_{21}}-1\right)\left(d_{14}\left(\alpha_{2}-2 E\left[\eta_{2} e^{\eta_{2}}\right]\right)+d_{12}\left(2 \alpha_{c}-2 E\left[\eta_{1} e^{\eta_{2}}\right]-\alpha_{1}\right)\right)
\end{aligned}
$$

Since coefficients of $v_{1}$ and $v_{2}$ are zero, $E[f(\eta)]=E[-2 \eta]$ or $d_{8}=d_{9}=d_{10}=0$. In addition, because $s_{11}$ and $s_{21}$ are arbitrary,

$$
\begin{aligned}
& 0=d_{13}\left(\alpha_{1}-2 E\left[\eta_{1} e^{\eta_{1}}\right]\right)+d_{11}\left(2 \alpha_{c}-2 E\left[\eta_{2} e^{\eta_{1}}\right]-\alpha_{2}\right) \\
& 0=d_{14}\left(\alpha_{2}-2 E\left[\eta_{2} e^{\eta_{2}}\right]\right)+d_{12}\left(2 \alpha_{c}-2 E\left[\eta_{1} e^{\eta_{2}}\right]-\alpha_{1}\right)
\end{aligned}
$$

Conditions of Equations (A47)-(A49) can be fulfilled with one of following five cases.

(1) If $d_{11}$ is not zero, for some constants $k_{1}$ and $k_{2}$, we have

$$
f_{c}\left(\eta_{1}, \eta_{2}\right)=\eta_{2} e^{\eta_{1}}+\frac{1}{2} f\left(\eta_{2}\right)+\frac{d_{13}}{2 d_{11}}\left(2 \eta_{1} e^{\eta_{1}}-f\left(\eta_{1}\right)\right)+k_{1}\left(e^{\eta_{1}}-1\right)+k_{2}\left(e^{\eta_{2}}-1\right) .
$$

Then $\frac{d_{13}}{2 d_{11}}\left(2 \eta_{1} e^{\eta_{1}}-f\left(\eta_{1}\right)\right)+k_{1}\left(e^{\eta_{1}}-1\right)=0$ and $\frac{1}{2} f\left(\eta_{2}\right)+k_{2}\left(e^{\eta_{2}}-1\right)=-\eta_{2}$ because $\frac{f_{c}(x, y)}{x y} \rightarrow 1$ as $(x, y) \rightarrow(0,0)$. Accordingly, $k_{2}=-1$ because $\frac{f(x)}{x^{2}} \rightarrow 1$ as $x \rightarrow 0$. This implies that $k_{1}=d_{13}=0$. Therefore, $f_{c}\left(\eta_{1}, \eta_{2}\right)=\eta_{2}\left(e^{\eta_{1}}-1\right), f(\eta)=2\left(e^{\eta}-\eta-1\right)$. Then, $d_{12}=d_{14}=0$.

(2) Similarly, if $d_{12}$ is not zero, $f_{c}\left(\eta_{1}, \eta_{2}\right)=\eta_{1}\left(e^{\eta_{2}}-1\right), f(\eta)=2\left(e^{\eta}-\eta-1\right)$ and $d_{11}=d_{13}=d_{14}=0$ Alternatively, when $d_{11}=d_{12}=0$, Equations (A47)-(A49) implies there are three more conditions as follows:

(3) $d_{11}=d_{12}=d_{13}=d_{14}=0, f(\eta)=2\left(e^{\eta}-\eta-1\right)$, with arbitrary function $f_{c}$.

(4) $d_{8}=d_{9}=d_{10}=d_{11}=d_{12}=0, f(\eta)=2\left(\eta e^{\eta}-e^{\eta}+1\right)$, with arbitrary function $f_{c}$.

(5) $\quad d_{8}=d_{9}=d_{10}=d_{11}=d_{12}=d_{13}=d_{14}=0$, with arbitrary functions $f$ and $f_{c}$.

(Proof for the second statement)

For the proof of the sufficiency of Equation (6) for the AP, we show that the function $g$ in Equation (6) satisfies the SAP (strong aggregation property): $E_{t}\left[g\left(X_{u}-X_{r}\right)\right]=E_{t}\left[g\left(X_{u}-X_{t}\right)\right]+E_{t}\left[g\left(X_{t}-X_{r}\right)\right]$, which is stronger condition than the $\mathrm{AP}$ of Equation (2). The $\mathrm{SAP}$ of the first seven terms in the equation is obvious. The 10th term is a generalization of the 8th and the 9th term and all these three terms do not vanish only if $f(\eta)=2\left(e^{\eta}-\eta-1\right)$. Thus, SAP of 10 th term implies the SAP of 8 th and 9 th terms. For convenience, let

$$
G_{u, t}=\phi_{1, u, t} \phi_{2, u, t}
$$

with 


$$
\phi_{i, u, t}=\left(V_{i, u}-V_{i, t}-2\left(s_{i, u}-s_{i, t}\right)\right) \quad \text { for } i \in\{1,2\}
$$

and

$$
V_{i, t} \equiv E_{t}\left[2\left(e^{s_{i, T}-s_{i . t}}-\left(s_{i, T}-s_{i . t}\right)-1\right)\right]=E_{t}\left[-2\left(s_{i, T}-s_{i . t}\right)\right]=E_{t}\left[V_{i, u}-2\left(s_{i, u}-s_{i, t}\right)\right]
$$
for $i \in\{1,2\}$.

Then, we have

$$
\begin{aligned}
E_{t}\left[G_{u, 0}\right] & =E_{t}\left[\left(\phi_{1, u, t}+\phi_{1, t, 0}\right)\left(\phi_{2, u, t}+\phi_{2, t, 0}\right)\right]=E_{t}\left[\phi_{1, u, t} \phi_{2, u, t}+\phi_{1, t, 0} \phi_{2, t, 0}\right] \\
& =E_{t}\left[G_{u, t}\right]+G_{t, 0} .
\end{aligned}
$$

Thus, we have the SAP of 8th and 9th terms as well as the SAP of 10th term.

Additionally, the SAP of the 11th term under the condition (1) implies that of the 12th term under the condition (2) and the 13th and 14th terms under the condition (4). Thus, we finish this proof by showing Equation (A55).

$$
E_{t}\left[F_{u, 0}\right]=E_{t}\left[F_{u, t}\right]+F_{t, 0}, \quad 0 \leq t \leq u \leq T
$$

where

$$
F_{u, t}=E_{t}\left[e^{s_{1, u}-s_{1 . t}}\left(\widehat{V}_{u}-\widehat{V}_{t}+2\left(s_{2, u}-s_{2 . t}\right)\right)\right]
$$

and

$$
\widehat{V}_{t} \equiv 2 E_{t}\left[\left(s_{2, T}-s_{2, t}\right)\left(e^{s_{1, T}-s_{1 . t}}-1\right)-\left(e^{s_{2, T}-s_{2 . t}}-\left(s_{2, T}-s_{2 . t}\right)-1\right)\right] .
$$

Equation (A57) is represented as:

$$
\begin{aligned}
\widehat{V}_{t} & =E_{t}\left[2 e^{s_{1, T}-s_{1 . t}}\left(s_{2, T}-s_{2, t}\right)\right] \\
& =E_{t}\left[2 e^{s_{1, T}-s_{1 . u}} e^{s_{1, u}-s_{1 . t}}\left(s_{2, T}-s_{2, u}\right)\right]+E_{t}\left[2 e^{s_{1, u}-s_{1 . t}}\left(s_{2, u}-s_{2, t}\right)\right] \\
& =E_{t}\left[e^{s_{1, u}-s_{1 . t}}\left(\widehat{V}_{u}+2\left(s_{2, u}-s_{2, t}\right)\right)\right] .
\end{aligned}
$$

It implies

$$
E_{t}\left[F_{u, t}\right]=E_{t}\left[e^{s_{1, u}-s_{1 . t}}\left(\widehat{V}_{u}+2\left(s_{2, u}-s_{2 . t}\right)\right)-e^{s_{1, u}-s_{1 . t}} \widehat{V}_{t}\right]=0
$$

and

$$
\begin{aligned}
E_{t}\left[F_{u, 0}\right] & =E_{t}\left[e^{s_{1, u}-s_{1.0}}\left(\widehat{V}_{u}-\widehat{V}_{0}+2\left(s_{2, u}-s_{2.0}\right)\right)\right] \\
& =E_{t}\left[e^{s_{1, u}-s_{1 . t}} e^{s_{1, t}-s_{1.0}}\left(\widehat{V}_{u}+2\left(s_{2, u}-s_{2 . t}\right)-\widehat{V}_{0}+2\left(s_{2, t}-s_{2.0}\right)\right)\right] \\
& =E_{t}\left[e^{s_{1, t}-s_{1.0}}\left(\widehat{V}_{t}-\widehat{V}_{0}+2\left(s_{2, t}-s_{2.0}\right)\right)\right]
\end{aligned}
$$

Due to Equations (A59) and (A60), Equation (A55) holds. 
Proof for Corollary 3.2

If a function is a realized ( $k, 1)$-comoment element for $k \in\{1,2\}$, Equation (11) should be decomposed as

$$
\begin{aligned}
g\left(\Delta s_{1}, \Delta s_{2}, \Delta v_{1}, \Delta v_{2}, \Delta v_{c}\right)= & \left(e^{\Delta s_{1}}-1\right) \phi_{1}\left(\Delta v_{1}, \Delta v_{2}, \Delta v_{c}\right)+\left(e^{\Delta s_{2}}-1\right) \phi_{2}\left(\Delta v_{1}, \Delta v_{2}, \Delta v_{c}\right) \\
& +g^{r}\left(\Delta s_{1}, \Delta s_{2}\right)
\end{aligned}
$$

such that $g^{r}\left(\Delta s_{1}, \Delta s_{2}\right)=O\left(\left(\Delta s_{1}\right)^{k} \Delta s_{2}\right)$ because of the restrictions $E\left[e^{\Delta s_{1}}\right]=1$ and $E\left[e^{\Delta s_{2}}\right]=1 .\left(\Delta v_{1}\right)^{2}$ cannot be a part of $\left(e^{\Delta s_{1}}-1\right) \phi_{1}\left(\Delta v_{1}, \Delta v_{2}, \Delta v_{c}\right)$ or $\left(e^{\Delta s_{2}}-1\right) \phi_{2}\left(\Delta v_{1}, \Delta v_{2}, \Delta v_{c}\right)$, as well as $g^{r}\left(\Delta s_{1}, \Delta s_{2}\right)$, because it is only in $h_{8}\left(\Delta v_{1}-2 \Delta s_{1}\right)^{2}$; thus, we have $h_{8}=0$. In a similar manner, by considering $\left(\Delta v_{2}\right)^{2}$ and $\Delta v_{1} \Delta v_{2}$, we have $h_{9}=h_{10}=0$. Accordingly, $e^{\Delta s_{1}} \Delta s_{2}$ and $e^{\Delta s_{2}} \Delta s_{1}$ are only cross-terms between $\Delta s_{1}$ and $\Delta s_{2}$. Therefore, none of condition (3), (4) or (5) can generate a realized ( $\left.k, 1\right)$-comoment element for $k \in\{1,2\}$.

Under the condition (1) in Proposition 3.1, $e^{\Delta s_{1}} \Delta s_{2}$ is the only cross-term between $\Delta s_{1}$ and $\Delta s_{2}$. In the remaining terms, we have $h_{5}=0, h_{6}=h_{11}$, and $h_{7}=-2 h_{11}$ to separate $\Delta v_{1}, \Delta v_{c}$, and $\Delta v_{2}$ from $g^{r}\left(\Delta s_{1}, \Delta s_{2}\right)$. Then, the remaining term $h_{1}\left(e^{\Delta s_{1}}-1\right)+h_{2} \Delta s_{1}+h_{3}\left(e^{\Delta s_{2}}-1\right)+h_{4} \Delta s_{2}+2 h_{11} e^{\Delta s_{1}} \Delta s_{2}$ is at most $O\left(\Delta s_{1} \Delta s_{2}\right)$ as $\left(\Delta s_{1}, \Delta s_{2}\right) \rightarrow(0,0)$ when $h_{1}=h_{2}=h_{3}=0$ and $h_{4}=-2 h_{11}$. It cannot be of $O\left(s_{1}^{2} s_{2}\right)$, and it is a realized $(1,1)$ comoment when $h_{4}=-1$.

In a similar manner, under condition (2), the function is at most is at most $O\left(s_{1} s_{2}\right)$, and it is a realized comoment when $h_{1}=h_{3}=h_{4}=h_{6}=h_{8}=h_{9}=h_{10}=0, h_{2}=h_{7}=-1, h_{5}=h_{12}=1 / 2$.

\section{Proof for Proposition 3.3}

(Proof for the first statement)

We use the common property A by omitting all terms related to the second security. Thus, $g$ is a function of $s_{1}, M_{2}$ and $M_{3}$ where $M_{2}=M_{2,0}$ and $M_{3}=M_{3,0}$. By integrating (A10) with respect to $M_{2}$ and $M_{3}$, we can obtain two different forms of the function $g$ :

$$
\begin{aligned}
g\left(s_{1}, M_{2}, M_{3}\right)= & a_{1,0} M_{2}+\left(b_{1,0} M_{2}+\frac{1}{2} b_{1,1} M_{2}^{2}+b_{1,4} M_{3} M_{2}\right)\left(e^{s_{1}}-1\right) \\
& +a_{1,3}\left(M_{2} f^{2}\left(s_{1}\right)+\frac{1}{2} M_{2}^{2}\right)+a_{1,6} M_{2}\left(f^{3}\left(s_{1}\right)+M_{3}\right)+g^{1}\left(s_{1}, M_{3}\right)
\end{aligned}
$$

and

$$
\begin{aligned}
g\left(s_{1}, M_{2}, M_{3}\right)= & a_{2,0} M_{3}+\left(b_{2,0} M_{3}+b_{2,1} M_{2} M_{3}+\frac{1}{2} b_{2,4} M_{3}^{2}\right)\left(e^{s_{1}}-1\right) \\
& +a_{2,3} M_{3}\left(f^{2}\left(s_{1}\right)+M_{2}\right)+a_{2,6}\left(f^{3}\left(s_{1}\right) M_{3}+\frac{1}{2} M_{3}^{2}\right)+g^{2}\left(s_{1}, M_{2}\right)
\end{aligned}
$$

with some functions $g^{1}$ and $g^{2}$. By combining (A62) and (A63), we obtain

$$
\begin{aligned}
g\left(s_{1}, M_{2}, M_{3}\right)= & a_{1} M_{2}+a_{2} M_{3} \\
& +\left(a_{3} M_{2}+a_{4} M_{3}+a_{5} M_{2}^{2}+a_{6} M_{2} M_{3}+a_{7} M_{3}^{2}\right)\left(e^{s_{1}}-1\right) \\
& +a_{8}\left(M_{2}^{2}+2 M_{2} f^{2}\left(s_{1}\right)\right)+a_{9}\left(M_{3}^{2}+2 f^{3}\left(s_{1}\right) M_{3}\right) \\
& +2 a_{10}\left(M_{2} M_{3}+f^{2}\left(s_{1}\right) M_{3}+f^{3}\left(s_{1}\right) M_{2}\right)+g^{s}\left(s_{1}\right)
\end{aligned}
$$

for some constants $a_{1}, \cdots, a_{10}$ and a function $g^{s}$ such that $g^{s}(0)=0$. Using the $\eta_{1}$ in Equation (A1), let us construct $\eta_{p}=\left\{\begin{array}{ll}\eta_{1} & \operatorname{Pr}=p \\ 0 & \operatorname{Pr}=1-p\end{array}\right.$ for a constant $p$ in $[0,1]$. Then, by substituting Equation (A64) into (A3) and replacing $\eta$ with $\eta_{p}$, we obtain: 
JDQS

30,2

$$
0=p\left(\begin{array}{l}
\left(e^{s_{1,1}}-1\right)\left(\begin{array}{l}
a_{3} \alpha_{2}+a_{4} \alpha_{3}-2 a_{5} m_{2} \alpha_{2} \\
+a_{6}\left(-m_{2} \alpha_{3}-m_{3} \alpha_{2}\right)-2 a_{7} m_{3} \alpha_{3}
\end{array}\right) \\
+2 a_{8}\left(\left(f^{2}\left(s_{1,1}+\eta_{1}\right)-f^{2}\left(s_{1,1}\right)-\alpha_{2}\right) m_{2}+\alpha_{2} f^{2}\left(s_{1,1}\right)\right) \\
+2 a_{9}\left(\left(f^{3}\left(s_{1,1}+\eta_{1}\right)-f^{3}\left(s_{1,1}\right)-\alpha_{3}\right) m_{3}+\alpha_{3} f^{3}\left(s_{1,1}\right)\right) \\
+2 a_{10}\left(\begin{array}{l}
\left(f^{2}\left(s_{1,1}+\eta_{1}\right)-f^{2}\left(s_{1,1}\right)-\alpha_{2}\right) m_{3}+\alpha_{3} f^{2}\left(s_{1,1}\right) \\
+\left(f^{3}\left(s_{1,1}+\eta_{1}\right)-f^{3}\left(s_{1,1}\right)-\alpha_{3}\right) m_{2}+\alpha_{2} f^{3}\left(s_{1,1}\right)
\end{array}\right) \\
-E\left[g^{s}\left(s_{1,1}+\eta_{1}\right)\right]+g^{s}\left(s_{1,1}\right)+E\left[g^{s}\left(\eta_{1}\right)\right] \\
+p^{2}\left(e^{s_{1,1}}-1\right)\left(a_{5} \alpha_{2}^{2}+a_{6} \alpha_{2} \alpha_{3}+a_{7} \alpha_{3}^{2}\right)
\end{array}\right)
$$

Because we can set $p$ arbitrary, coefficients of $p$ and $p^{2}$ are zero. Therefore, we have

$$
a_{5} \alpha_{2}^{2}+a_{6} \alpha_{2} \alpha_{3}+a_{7} \alpha_{3}^{2}=0
$$

and

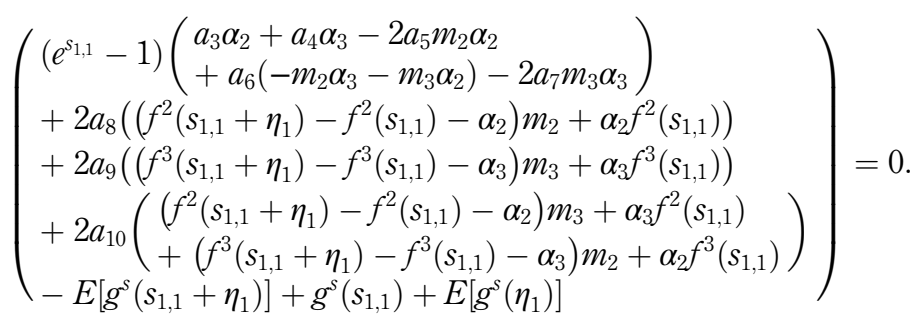

Equation (A66) implies that

$$
a_{5}=a_{6}=a_{7}=0
$$

because $\eta_{1}$ is an arbitrary random variable with $E\left[e^{\eta_{1}}\right]=1$. Also, in Equation (A67), coefficients of $m_{2}$ and $m_{3}$ are zero because we can set arbitrary values for them. Thus, we have:

$$
\begin{aligned}
& 0=a_{8}\left(f^{2}\left(s_{1,1}+\eta_{1}\right)-f^{2}\left(s_{1,1}\right)-\alpha_{2}\right)+a_{10}\left(f^{3}\left(s_{1,1}+\eta_{1}\right)-f^{3}\left(s_{1,1}\right)-\alpha_{3}\right) \\
& 0=a_{9}\left(f^{3}\left(s_{1,1}+\eta_{1}\right)-f^{3}\left(s_{1,1}\right)-\alpha_{3}\right)+a_{10}\left(f^{2}\left(s_{1,1}+\eta_{1}\right)-f^{2}\left(s_{1,1}\right)-\alpha_{2}\right)
\end{aligned}
$$

There are three cases that satisfy both (A69) and (A70). We call them condition A.1 as follows:

\section{Condition A.1}

(1) $\exists f^{3}$ such that $\forall\left(s_{1}, \eta_{1}\right), f^{3}\left(s_{1,1}+\eta_{1}\right)-f^{3}\left(s_{1,1}\right)-\alpha_{3}=0$ and $a_{8}=a_{10}=0$,

(2) $\exists\left(a, f^{2}, f^{3}\right)$ such that $\forall\left(s_{1,1}, \eta_{1}\right), f^{2}\left(s_{1,1}+\eta_{1}\right)-f^{2}\left(s_{1,1}\right)-\alpha_{2}+a\left(f^{3}\left(s_{1,1}+\eta_{1}\right)-f^{3}\left(s_{1,1}\right)-\alpha_{3}\right)$ $=0$ with $a_{10}=a_{8} a$ and $a_{9}=a^{2} a_{8}$,

(3) $a_{8}=a_{9}=a_{10}=0$.

First, we check the condition in A.1.(1). Substituting $\eta_{1}=\left\{\begin{array}{ll}\ln (1+k), & \operatorname{Pr}=0.5 \\ \ln (1-k), & \operatorname{Pr}=0.5\end{array}\right.$ into $\frac{2}{k^{2}}\left(f^{3}\left(s_{1,1}+\eta_{1}\right)-f^{3}\left(s_{1,1}\right)-\alpha_{3}\right)=0$, using $\lim _{x \rightarrow 0} \frac{f^{3}(x)}{x^{3}}=1$, and taking the limit for $k \rightarrow 0$ yield:

$$
\left(f^{3}\right)^{\prime \prime}\left(s_{1,1}\right)-\left(f^{3}\right)^{\prime}\left(s_{1,1}\right)=0
$$


Thus, $f^{3}\left(s_{1,1}\right)=b_{1} e^{s_{1,1}}+b_{2}$ for some constants $b_{1}$ and $b_{2}$. However, there are no $b_{1}$ and $b_{2}$ that makes $\lim _{x \rightarrow 0} \frac{f^{3}(x)}{x^{3}}=1$. Therefore, condition A.1 (1) is impossible.

Second, let us check condition A.1.(2). Substituting $f^{a}(x)=f^{2}(x)+a f^{3}(x)$ and $\eta_{1}=$ $\left\{\begin{array}{ll}\ln (1+k), & \operatorname{Pr}=0.5 \\ \ln (1-k), & \operatorname{Pr}=0.5\end{array}\right.$ into $f^{2}\left(s_{1,1}+\eta_{1}\right)-f^{2}\left(s_{1,1}\right)-\alpha_{2}+a\left(f^{3}\left(s_{1,1}+\eta_{1}\right)-f^{3}\left(s_{1,1}\right)-\alpha_{3}\right)=0$, using $\lim _{x \rightarrow 0} \frac{f^{2}(x)}{x^{2}}=1$ and $\lim _{x \rightarrow 0} \frac{f^{3}(x)}{x^{3}}=1$, and taking the limit for $k \rightarrow 0$ yield:

$$
\left(f^{a}\right)^{\prime \prime}\left(s_{1,1}\right)-\left(f^{a}\right)^{\prime}\left(s_{1,1}\right)-2=0
$$

Thus, we have $f^{a}\left(s_{1,1}\right)=b_{1} e^{s_{1,1}}+b_{2}-2 s_{1,1}$ for some constants $b_{1}$ and $b_{2}$. Because of the conditions $\lim _{x \rightarrow 0} \frac{f^{2}(x)}{x^{2}}=1$ and $\lim _{x \rightarrow 0} \frac{f^{3}(x)}{x^{3}}=1, f^{2}$ has the following form:

$$
f^{2}(s)=2\left(e^{s}-s-1\right)-a f^{3}(s) .
$$

Substituting (A68), (A73), and Condition A.1 (2) into (A67) yields:

$$
\begin{aligned}
E\left[g^{s}\left(s_{1,1}+\eta_{1}\right)\right]-g^{s}\left(s_{1,1}\right)-E\left[g^{s}\left(\eta_{1}\right)\right]= & \left(e^{s_{1,1}}-1\right)\left(a_{3} \alpha_{2}+a_{4} \alpha_{3}\right) \\
& +4 a_{8}\left(\alpha_{2}+a \alpha_{3}\right)\left(e^{s_{1,1}}-s_{1,1}-1\right)
\end{aligned}
$$

Equation (A74) with $a_{8}=0$ satisfies (A67) with (A68) and condition A.1 (3). Therefore, Equation (A74) is a general equation for $g^{s}$. Again, by letting $\eta_{1}=\left\{\begin{array}{ll}\ln (1+k), & \operatorname{Pr}=0.5 \\ \ln (1-k), & \operatorname{Pr}=0.5\end{array}\right.$ and taking the limit, we can obtain a differential equation:

$$
\left(g^{s}\right)^{\prime}-\left(g^{s}\right)^{\prime \prime}+2 a_{3}\left(e^{s}-1\right)+8 a_{8}\left(e^{s}-s-1\right)=\text { const. }
$$

Using $g^{s}(0)=0, g^{s}$ is represented as follows:

$$
g^{s}(s)=a_{9} s+a_{10}\left(e^{s}-1\right)+4 a_{8} s^{2}+\left(8 a_{8}+2 a_{3}\right) s e^{s}
$$

with additional constants $a_{9}$ and $a_{10}$. Substituting it into (A64) yields:

$$
\begin{aligned}
g\left(s, M_{2}, M_{3}\right)= & a_{1} M_{2}+a_{2} M_{3}+\left(a_{3} M_{2}+a_{4} M_{3}\right)\left(e^{s}-1\right)+a_{8}\left(M_{2}+a M_{3}-2 s\right)^{2} \\
& +4 a_{8}\left(M_{2}+a M_{3}\right)\left(e^{s}-1\right)+a_{9} s+a_{10}\left(e^{s}-1\right)+\left(8 a_{8}+2 a_{3}\right) s e^{s}
\end{aligned}
$$

or

$$
\begin{aligned}
g\left(s, M_{2}, M_{3}\right)= & d_{1} M_{2}+d_{2} M_{3}+d_{3} M_{3} e^{s}+d_{4}\left(M_{2}+a M_{3}-2 s\right)^{2}+d_{5}\left(M_{2}+a M_{3}+2 s\right) e^{s} \\
& +d_{6} s+d_{7}\left(e^{s}-1\right)
\end{aligned}
$$

where $d_{1}=a_{1}-a_{3}, d_{2}=a_{2}-a_{4}, d_{3}=a_{4}-a a_{3}, d_{4}=a_{8}, d_{5}=a_{3}+4 a_{8}, d_{6}=d_{9}$ and $d_{7}=d_{10}$. Then, substituting these into (A3) yields

$$
\begin{aligned}
& d_{4}\left(4 s_{1,1}+2\left(m_{2}+a m_{3}-\alpha_{2}-a \alpha_{3}\right)\right)\left(E\left[2 \eta_{1}\right]+\alpha_{2}+a \alpha_{3}\right)+\left(e^{s_{1,1}}-1\right)\left(d _ { 5 } \left(E\left[2 \eta_{1} e^{\eta_{1}}\right]\right.\right. \\
& \left.\left.\quad-\alpha_{2}-a \alpha_{3}\right)-d_{3} \alpha_{3}\right)=0
\end{aligned}
$$

Because $s_{1}$ is arbitrary, we have the following cases.

\section{Condition A.2}
(1) $d_{3}=d_{4}=d_{5}=0$
(2) $d_{3}=d_{5}=0$ and $E\left[2 \eta_{1}\right]+\alpha_{2}+a \alpha_{3}=0$ 
JDQS

30,2

112

(3) $d_{4}=0$ and $E\left[2 \eta_{1} e^{\eta_{1}}\right]-\alpha_{2}-h \alpha_{3}$ with $h=a+d_{3} / d_{5}$.

Recall that $\mathrm{E}\left[e^{\eta_{1}}-1\right]=0, \alpha_{k}=E\left[f^{k}\left(\eta_{1}\right)\right]$ and $\frac{f^{2}\left(\eta_{1}\right)+a f^{3}\left(\eta_{1}\right)}{\eta_{1}^{2}} \rightarrow 1$ for $\eta_{1} \rightarrow 0$. Therefore, when condition A.2 (2) holds, we obtain $f^{2}(\Delta s)+a f^{3}(\Delta s)=2\left(e^{\Delta s}-n_{1}^{1} \Delta s-1\right)$. Next, condition A.2 (3) is equivalent to $d_{3}=d_{4}=0$ with

$$
E\left[2 \eta_{1} e^{\eta_{1}}\right]-\alpha_{2}-a \alpha_{3}=0
$$

which implies that

$$
f^{2}(\Delta s)+a f^{3}(\Delta s)=2\left(\Delta s e^{\Delta s}-e^{\Delta s}+1\right) .
$$

Rearranging the above equations yields the equation and the condition of Proposition 3.1. This implies that Equation (14) is a candidate for a function with the aggregation property.

(Proof for the second statement)

Similar to Proposition 3.3, it is enough to show the SAP of Equation (14) holds. The SAP of the first four terms in the equation is obvious. Proofs for the 5 th and 6 th terms in this proposition are similar to those of the 10th and 11th terms in Proposition 3.1, respectively.

\section{Appendix 2}

Proof for Proposition 4.2

Let us set $S_{t}=S_{1, t}+a S_{2, t}$ and $X_{t}=\left(M_{t}^{(1)}, \ldots, M_{t}^{(L-1)}, 0\right)$ with $M_{t}^{(l)}={ }_{{ }_{{ }_{\kappa_{l}}}}\left(S_{T}\right)$. Then, according to Fukasawa and Matsushita (2021),

$$
E_{0}\left[\sum_{j=1}^{N} B_{L}\left(X_{t_{j}}-X_{t_{j-1}}\right)\right]=E_{0}\left[B_{L}\left(X_{T}-X_{0}, 0\right)\right]=\kappa_{L}^{0}\left(S_{T}\right) .
$$

According to Equation (18), $M_{t}^{(l)}$ is decomposed to $\sum_{k=0}^{l} a^{k}\left(\begin{array}{l}l \\ k\end{array}\right) M_{t}^{(l-k, k)}$ for $M_{t}^{(l-k, k)}=$ ${ }^{t} \kappa_{l-k, k}\left(S_{1, T}, S_{2, T}\right)$. In other words, the right hand side of Equation (A82) can be represented as

$$
\sum_{k=0}^{L} a^{k}\left(\begin{array}{l}
L \\
k
\end{array}\right){ }^{0} \kappa_{L-k, k}\left(S_{1, T}, S_{2, T}\right) .
$$

For convenience, we denote the summand of the left hand side of Equation (A82) as $B_{L}(\Delta X)$. By the definition of $B_{L}$, we can arrange it as follows.

$$
\begin{aligned}
B_{L}(\Delta X) & =\left.\frac{\partial^{L}}{\partial u^{L}}\left(\exp \left(\sum_{i=1}^{L-1} \Delta M^{(i)} \frac{u^{i}}{i !}\right)\right)\right|_{u=0}=\left.\frac{\partial^{L}}{\partial u^{L}}\left(\exp \left(\sum_{i=1}^{L-1} \sum_{j=0}^{i} a^{j}\left(\begin{array}{l}
i \\
j
\end{array}\right) \Delta M^{(i-j, j)} \frac{u^{i}}{i !}\right)\right)\right|_{u=0} \\
& =\left.\frac{\partial^{L}}{\partial u^{L}}\left(\exp \left(\sum_{i_{0}=1}^{L-1} \Delta M^{\left(i_{0}, 0\right)} \frac{u^{i_{0}}}{i_{0} !}+a \sum_{i_{1}=1}^{L-1} i_{1} \Delta M^{\left(i_{1}-1,1\right)} \frac{u^{i_{1}}}{i_{1} !}+O\left(a^{2}\right)\right)\right)\right|_{u=0} \\
& =\left.\frac{\partial^{L}}{\partial u^{L}}\left(\exp \left(\sum_{i_{0}=1}^{L-1} \Delta M^{\left(i_{0}, 0\right)} \frac{u^{i_{0}}}{i_{0} !}\right)\right)\right|_{u=0} \\
& +\left.a \sum_{i_{1}=1}^{L-1}\left(\begin{array}{c}
L \\
i_{1}
\end{array}\right) i_{1} \Delta M^{\left(i_{1}-1,1\right)} \frac{\partial^{L-i_{1}}}{\partial u^{L-i_{1}}}\left(\exp \left(\sum_{i_{0}=1}^{L-1} \Delta M^{\left(i_{0}, 0\right)} \frac{u^{i_{0}}}{i_{0} !}\right)\right)\right|_{u=0}+O\left(a^{2}\right) \\
& =B_{L}\left(\Delta M^{(1,0)}, \cdots, \Delta M^{(L-1,0)}, 0\right) \\
& +a L \sum_{i_{1}=1}^{L-1}\left(\begin{array}{c}
L-1 \\
i_{1}-1
\end{array}\right) \Delta M^{\left(i_{1}-1,1\right)} B_{L-i_{1}}\left(\Delta M^{(1,0)}, \cdots, \Delta M^{\left(L-i_{1}, 0\right)}\right)+O\left(a^{2}\right)
\end{aligned}
$$


Because $a$ is arbitrary, the coefficient of $a$ of the left hand side of Equation (A82) is equal to the coefficient of $a$ of the right hand side of Equation (A82). Therefore, by Equations (A82), (A83) and (A84), we have

$$
{ }^{0} \kappa_{L-1,1}\left(S_{1, T}, S_{2, T}\right)=E_{0}\left[\sum_{j=1}^{N} \sum_{i_{1}=1}^{L-1}\left(\begin{array}{c}
L-1 \\
i_{1}-1
\end{array}\right) \Delta M^{\left(i_{1}-1,1\right)} B_{L-i_{1}}\left(\Delta M^{(1,0)}, \Delta M^{(2,0)}, \cdots, \Delta M^{\left(L-i_{1}, 0\right)}\right)\right] .
$$

Geometric and arithmetic comoments

(A85)

\section{Corresponding author}

Kwangil Bae can be contacted at: k.bae@chonnam.ac.kr

For instructions on how to order reprints of this article, please visit our website: www.emeraldgrouppublishing.com/licensing/reprints.htm Or contact us for further details: permissions@emeraldinsight.com 